\title{
EL RÉGIMEN JURÍDICO DE LOS MEDIOS DE COMUNICACIÓN EN EL REINO UNIDO
}

\author{
YOLANDA FERNÁNDEZ VIVAS \\ Profesora Contratada Doctora de Derecho Constitucional \\ Universidad de Alcalá
}

\author{
SUMARIO \\ I. Introducción. \\ II. Prensa. \\ III. Radiotelevisión pública. \\ IV. Radiotelevisión privada. \\ V. Las autoridades de control.
}

\section{INTRODUCCIÓN}

El régimen jurídico de los medios de comunicación en el Reino Unido se caracteriza, en primer lugar, por la ausencia de un marco legal único, algo que, por otra parte, no es de extrañar tratándose éste de un sistema de common law. Esta situación se agrava más, si cabe, en los últimos años, debido a la importante transformación que está sufriendo el sector audiovisual, que permite acceder a distintos medios mediante diversos dispositivos electrónicos, por lo que una de las cuestiones que está debatiéndose hoy en día es sobre la idoneidad de mantener una estructura reguladora por sectores (televisión, internet, telecomunicaciones) o bien fijar un marco legal único ${ }^{1}$.

No obstante, y a pesar de la inexistencia de un marco legal único en el Reino Unido, existe una tradición de debate constitucional en torno a los objetivos de la televisión pública y a la relación de ésta su relación con el Estado y con la au-

1 B. Belando Garín, «Democracia y medios de comunicación. Cuestiones al hilo de la reforma de los medios de comunicación en el Reino Unido», en A. Boix PALOP/J. M. VIDAL Beltrán, La nueva regulación del audiovisual: medios, derechos y libertades, Thomson Reuters - Aranzadi, Pamplona, 2014, p. 122. 
diencia, en el que participan tanto el Gobierno como los políticos y los profesionales del medio, que ha contribuido a formar una especie de «consenso constitucional» sobre cuáles son las principales razones para regular la televisión y el marco de actuación en este ámbito, y que ha permitido mantener a la radiotelevisión pública como un ente independiente y ajeno a los cambios gubernamentales y políticos ${ }^{2}$.

En segundo lugar, otra de las características más relevantes del régimen jurídico de los media en el Reino Unido es la tendencia a la autorregulación, esto es, al establecimiento de reglas de funcionamiento y de organización por parte de los propios medios y que quedan sometidos a supervisión por parte de organismos de control, tanto públicos como privados ${ }^{3}$. En este sentido, y a pesar de que la regla general es incentivar la autorregulación, hay sectores, como la radiotelevisión, en las que la autorregulación se utiliza menos y existe una mayor intervención pública en la definición de sus objetivos y en el establecimiento de su organización y funcionamiento ${ }^{4}$.

\section{PRENSA}

La prensa escrita se rige por los principio de libertad y de autorregulación. En primer lugar, y desde una perspectiva totalmente liberal, el sector de la prensa tiene como principio esencial la garantía de las libertades de expresión e información. De este modo, no existe ningún tipo de control previo y se reconoce la libertad de creación de medios de comunicación escritos sin necesidad de tener que solicitar autorización para ello. No obstante, y a pesar de que no existe ninguna norma que fije la creación, organización, funciones o financiación de la prensa, sí que hay diferentes normas que son de aplicación a este sector, todas ellas relacionadas con los contenidos ${ }^{5}$.

2 E. Barendt, Broadcasting Law. A comparative study, Clarendon Press, Oxford, 1993, p. 51 y ss.; J. MorGAN, «The BBC and the concept of public service broadcasting», en C. MCCABE/O. STEwART (eds.), The BBC and Public Service Broadcasting, 1986; T. GibBons, «La televisión pública en el Reino Unido», en J. J. GonZÁlez Encinar (ed.), La televisión pública en la Unión Europea, McGraw Hill, Madrid, 1996, p. 28; T. Gibbons, Regulating the Media, Sweet \& Maxwell, Londres, 1991, 1. a ed., p. 32 y ss.

3 W. SCHulz/T. Held, Regulated self-regulation as a form of modern Government. Analysis of case studies from media and telecommunications law, Luton University Press, Eastleigh, 2004, p. 7; S. NikOLTCHEv, "Co-regulation of the Media in Europe: European provisions for the establishment of co-regulation framework», en European Audiovisual Observatory, Iris Plus Collection: Key Legal Questions for the Audiovisual sector, Estrasburgo, 2003, pp. 7 a 16.

4 B. Belando Garín, «Democracia y medios de comunicación. Cuestiones al hilo de la reforma de los medios de comunicación en el Reino Unido», op. cit., pp. 123 y 128.

5 Varias son las leyes que regulan los límites que se imponen a la libertad de expresión en defensa de otros valores o intereses legítimos, así, existen leyes sobre difamación (Defamation Act 2013), contra las publicaciones que incitan al odio racial y al discurso del odio (arts. 17 y ss. Public Order Act 1986), sobre las noticias relativas a menores implicados en juicios (Children and Young Persons Act 2008), sobre derechos de autor (Co- 
En segundo lugar, la prensa se rige por el principio de autorregulación. El sistema actual de autorregulación de la prensa tiene su origen en la primera mitad del siglo xx, cuando la Unión Nacional de Periodistas adoptó su código de conducta y se creó una Comisión que propuso la creación de un Consejo General de la Prensa que se encargase de supervisar la creciente concentración en los medios escritos, fijar estándares éticos tanto para los propietarios de los periódicos como para los periodistas y analizar el impacto de las diferentes políticas editoriales $^{6}$. De este modo, en 1953, se creó el Consejo General de la Prensa, que estaba formado en su totalidad por profesionales de la industria de la prensa, y era financiado por este sector pero que carecía de poderes vinculantes. Este consejo se transformó años después en el British Press Council y posteriormente en la Press Complaints Commission ${ }^{7}$.

No obstante, el escándalo de las escuchas telefónicas y otras prácticas periodísticas ilegales realizadas por el grupo de comunicación News of the World, propiedad del magnate de la comunicación Rupert Murdoch, durante 2011, puso de manifiesto la debilidad del marco jurídico en el que se desarrollaba la actividad periodística en el Reino Unido, así como la necesidad de establecer mayores controles y exigir responsabilidades a los profesionales tanto respecto a la calidad de la información suministrada como a la forma en la que ésta era conseguida. Como resultado de todo ello, el Parlamento creó una Comisión de investigación, que finalizó su labor en 2012, y en cuyo informe se proponía un nuevo sistema de supervisión para la prensa escrita, con mayores poderes de supervisión y control ${ }^{8}$. Esta propuesta se ha materializado en la creación de un nuevo organismo de control, la Independent Press Standards Organisation (IPSO).

\section{RADIOTELEVISIÓN PÚBLICA}

La televisión comenzó a emitir en el Reino Unido a mediados de los años treinta y desde sus inicios se configuró conforme a la idea de «servicio público» tal y como éste se había desarrollado ya antes para la radio. Esa tradición de «ser-

pyright, Designs and Patents Act 1988), sobre secretos oficiales (Official Secrets Act 1989) y sobre aspectos relacionados con el terrorismo (The Prevention of Terrorism Act 2005).

6 R. Cohen-Almagor, Speech, Media and Ethics. The Limits of Free Expression, Palgrave, Hampshire, 2001, pp. 124 y 125; G. Murray, The Press and the Public, Southern Illinois University Press, Carbondale and Edwardsville, 1972, pp. 8 a 27.

7 S. RAGONE, «La autorregulación de los medios de comunicación en el Reino Unido», en J. C. DAVARA De CARA/J. De Miguel BÁRCena (eds.), La autorregulación de los medios de comunicación como sistema de control, 2014, Bosch, Barcelona, pp. 137 a 139.

8 La Comisión de Investigación conocida como «the Leveson Inquiry», comenzó sus trabajos el 13 de julio de 2011. Para poder acceder a la toda la información y el procedimiento llevado a cabo por esta Comisión, vid., http://webarchive.nationalarchives.gov.uk/20140122145147/http:/www.levesoninquiry.org.uk/. B. BELANDO GARín, «Democracia y medios de comunicación. Cuestiones al hilo de la reforma de los medios de comunicación en el Reino Unido», op. cit., p. 122. 
vicio público» ha definido al sistema de radiotelevisión británico desde sus comienzos y ha servido como referente y modelo para los sistemas de radiotelevisión pública que se desarrollaron más tarde en Europa9 .

Aunque la idea de servicio público ha estado sujeta a diversas interpretaciones, y ha ido evolucionando en las últimas décadas, principalmente por los nuevos cambios tecnológicos que se han producido en materia audiovisual, existe un contenido básico generalmente aceptado ${ }^{10}$. Las características que pueden predicarse del servicio público son la universalidad, la independencia editorial y la financiación pública. La universalidad, es entendida, por un lado, como exigencia de que todos los programas lleguen a toda la población y, por otro lado, como exigencia de que el contenido de los programas esté pensado para todos los intereses y gustos. Por su parte, la independencia editorial se predica respecto de cualquier tipo de presión o interés tanto comercial como gubernamental, especialmente, la independencia frente a la política y al Gobierno. Este principio refleja parcialmente un concepto más amplio, el de la libertad de la comunicación, pero responde también a la idea de que un servicio dedicado al público no debe estar dominado por intereses particulares. Finalmente, la utilización de recursos públicos para financiar o subvencionar los programas; en este caso, la televisión se concibe como un bien público ofrecido por el Estado. Ciertamente, la financiación pública no es una condición necesaria para que la televisión sea pública, pero no es una casualidad que la desconfianza general en el mercado, como mecanismo apropiado para lograr los objetivos del servicio público, haya sido habitual entre los profesionales del medio ${ }^{11}$.

La segunda característica, llamativa, de la radiotelevisión en el Reino Unido es que ni sus objetivos ni su actuación han estado tradicionalmente fijados

9 T. GibBons, Regulating the Media, 1998 (2. ${ }^{a}$ ed.), Sweet \& Maxwell, Londres, p. 55 y ss.; T. GIBBONs, «La televisión pública en el Reino Unido», op. cit., p. 27 y pp. 30 a 35; T. Prosser, «United Kingdom», en European Audiovisual Observatory, The Public Service Broadcasting Culture, Estrasburgo, 2007, p. 103.

10 Sobre el concepto y evolución del servicio público de radiotelevisión vid., D. ATKINson, «Legitimacy of public television in the era of the market», en D. AtKinson/M. RABOY, Public Service Broadcasting: the challenges of the twenty-first Century, UNESCO Publishing, 1997, pp. 40 a 42; BROADCASTING RESEARCH UNIT, The Public Service Idea in British Broadcasting, 1985; R. CRAUFURD SMITH, Broadcasting Law and Fundamental Rights, Oxford University Press, Oxford, 1997, p. 46; T. GibBons, Regulating the Media, op. cit., pp. 55 a 64; J. Harrison/L. Woods, European Broadcasting Law and Policy, Cambridge University Press, Cambridge, 2007, pp. 31 a 33; OFCOM, Review of Public Service Television Broadcasting, de 25 de abril de 2004, pp. 18 a 23, disponible en http://www.ofcom.org.uk/consult/condocs/psb/psb/psb.pdf. Por otra parte, llama la atención que a pesar de los nuevos avances tecnológicos, en virtud de los cuales ya no existe escasez de frecuencias — una de las razones para la consideración del servicio público— todavía se sigue considerando el concepto del «public service broadcasting» como un elemento clave en el futuro digital de la comunicación, llamado a jugar un papel incluso de mayor importancia que en el pasado analógico.

11 S. Buckley/K. Duer/T. Mendel/S. O. Siochru, Broadcasting, Voice and Accountability. A Public Interest Approach to Policy, Law and Regulation, The World Bank Institute, Washington, 2008, pp. 38 y 39; T. GibBons, «La televisión pública en el Reino Unido», op. cit., p. 31; N. GARnHAM, «The broadcasting market and the future of the BBC», en The Political Quarterly, 65 (1994), p. 11. 
en un marco legal único y escrito ${ }^{12}$. Sin embargo, y como ya hemos indicado, a pesar de la inexistencia de un marco legal único en el Reino Unido, existe una tradición de debate constitucional en torno a los objetivos de la televisión pública y a la relación de ésta su relación con el Estado y con la audiencia, en el que participan tanto el Gobierno como los políticos y los profesionales del medio. De este modo, este debate ha ayudado a formar una especie de «consenso constitucional» sobre cuáles son las principales razones para regular la televisión y el marco de actuación en este ámbito. Por este motivo, hasta ahora, el tratamiento de la radiotelevisión pública se caracterizaba por una considerable discrecionalidad, tanto de los reguladores como de los gestores a los que se había confiado la administración independiente de los intereses de la televisión pública. Sin embargo, en los últimos años se ha apreciado signos de debilitamiento de esa forma de consenso constitucional, y esto se ha unido al hecho de que se han ido aprobando cada vez más normas jurídicas que, bien de forma directa, bien de forma indirecta, afectan a la organización y funcionamiento de la BBC. Por este motivo, un sector doctrinal cada vez más amplio exige que se aclare el marco legal de la BBC mediante la aprobación de una ley formal en la que se establezca claramente y de forma estable todo lo relacionado con su estatuto orgánico y funciones ${ }^{13}$.

En la actualidad, en el Reino Unido el servicio público de radiotelevisión se presta por tres entes: la British Broadcasting Corporation (BBC), de difusión nacional y con contenidos generalistas, la Welsh Authority, el servicio de radiotelevisión propio de la región de Gales, y Channel 4, un servicio de radiotelevisión pública especializado por sus contenidos.

\section{La British Broadcasting Corporation}

\section{A) Naturaleza}

a) Creación y estatuto jurídico

La British Broadcasting Corporation (BBC) se creó originalmente en 1922, como una empresa privada, la British Broadcasting Company. Pocos años después, concretamente, el 20 de diciembre de 1926, el Rey Jorge V otorgó a la BBC una

12 Sí resulta paradójico, al menos, que las televisiones privadas, que en buena medida también desarrollan obligaciones de servicio público como la BBC, se hayan creado por ley, mientras que la BBC no tiene una ley formal que establezca su regulación.

13 E. Barendt, Broadcasting Law. A comparative study, op. cit., p. 51 y ss.; J. MorGAN, «The BBC and the concept of public service broadcasting», en C. Mccabe/O. STEWART (eds.), The BBC and Public Service Broadcasting, 1986; T. GibBons, «La televisión pública en el Reino Unido», op. cit., p. 28; T. GibBons, Re-

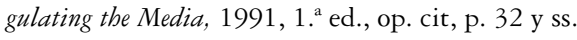


Carta Real de fundación, la Royal Charter ${ }^{14}$. A partir de entonces, la BBC se ha regulado por sucesivas «Cartas» de esa naturaleza que se renuevan cada diez o quince años ${ }^{15}$. En dicha Carta se especifican las obligaciones de servicio público, la organización interna de la BBC y su forma de financiación.

La Royal Charter se concede por un período que oscila entre los diez y quince años. La Carta actual se aprobó el 16 de julio de 2006, entró en vigor el 1 de enero de 2007 y es válida hasta el 31 de diciembre de $2016^{16}$. En ella se define a la BBC como una corporación pública, se establecen las obligaciones de servicio público y la organización interna de la compañía ${ }^{17}$. En el proceso de elaboración de la actual Royal Charter se produjo un intenso debate político y académico, que ha provocado una profunda transformación de la BBC, tanto desde el punto de vista organizativo, como también desde la perspectiva técnica, con el fin de adaptar a la Compañía a los nuevos desafíos de la tecnología digital ${ }^{18}$.

14 En el momento de fundación de la BBC se optó porque su régimen jurídico se regulara a través de una norma jurídica poco habitual, una Royal Charter, o Carta Real, y no por una ley específica. Los motivos por los que se tomó esta decisión fueron los siguientes: primero, porque una Carta Real permitiría a la BBC mayor libertad de movimientos que si se le sometía al régimen legal vigente en ese momento; segundo, porque se entendía que la posibilidad de crear una ley específica hubiera comprometido la independencia de la nueva Corporación, al asociarla, a ojos de los ciudadanos, con el Parlamento y el proceso político; y tercero, porque con una Carta Real, en teoría, se le se concedía mayor dignidad y estatus a la nueva BBC. Sin embargo, además de las posibles ventajas que proporcionaba la utilización de esta figura normativa tan poco usual, el haber recurrido a una Carta Real para crear la BBC tuvo ciertos inconvenientes, como es el hecho de que se concede al Gobierno un considerable poder discrecional para decidir sobre la manera concreta en que esta institución va a funcionar, ya que, aunque la Royal Charter forma parte del ejercicio de las prerrogativas reales (poder residual del rey o de la reina en el common law, de naturaleza ejecutiva), ese poder o potestad real es meramente formal, porque en realidad buena parte del poder que el monarca ejerce es controlado de facto por el Gobierno. Por ello, un amplio sector de la doctrina ha abogado por la adopción de un marco legal estable y formal para la BBC. Sin embargo, hasta ahora, el Gobierno británico ha rechazado estas propuestas, al considerar que mediante una Carta Real se asegura mejor la independencia de la BBC. Vid. Vid. House or Lords Select Committee on the BBC Charter Review, "The Review of the BBC's Royal Charter», HL 50, 2005-06; A. BRIGGs, The History of Broadcasting in the United Kingdom, Vol. I: The birth of broadcasting, op. cit., p. 352; A. W. BRADLEY/K. W. EwING, Constitutional and Administrative Law, $14 .^{\circ}$ ed., Ed. Longman, Harlow, 2006, p. 547. E. BARENDT, «Legal aspects of BBC Charter renewal», en Political Quarterly, n. ${ }^{\circ}$ 65, 1994, pp. 8-20; T. Prosser, «United Kingdom», en European Audiovisual Observatory, The Public service Broadcasting Culture, Estrasburgo, 2007, p. 103; T. GibBons, «La televisión pública en el Reino Unido», op. cit., pp. 36 a 38.

15 Sobre el origen y evolución de la BBC, véanse los estudios de A. BRIGgs, The History of Broadcasting in the United Kingdom, Oxford University Press, Oxford, 1961; T. Burns, The BBC: Public Institution and Private World, MacMillan, Edimburgo, 1977; A. BRIGGS, The BBC: the first fifty years, Oxford University Press, Oxford, 1984; P. Scannell/D. Cardiff, A Social History of Broadcasting, Blackwell, Oxford, 1991; R. H. Coarse, British Broadcasting: A Study in Monopoly, Longman, Essex, 1950, p. 15; E. BArendT/L. HitCHens, Media Law. Cases and materials, Longman, Essex, 2000, p. 70.

16 Royal Charter, art. 2.

17 P. Humphreys, «Das Rundfunksystem Großbritanniens», en VVAA, Internationales Handbuch für Hörfunk und Fernsehen, 1998-1999, Hans Bredow Institut, Nomos, Baden Baden, 2000 p. 346; P. CAREY, Media Law, Sweet \& Maxwell, London, 1999 (2. ed.), p. 205.

18 La elaboración de la actual Royal Charter ha sido especialmente participativa, no sólo en la Comisión parlamentaria encargada de su elaboración, sino también con la presentación de distintos documentos e informes por parte de diversos colectivos incluida la OFCOM. Para acceder a todos los documentos presentados para abordar el futuro de la BBC y la elaboración de la actual Royal Charter, vid. http://www.bbccharterre- 
Junto con la Royal Charter, la otra norma que establece el régimen jurídico de la $\mathrm{BBC}$ es la Licence and Agreement (literalmente, Licencia y Convenio) ${ }^{19}$, ya que la BBC está obligada a solicitar del Gobierno una licencia para transmitir y queda vinculada por las condiciones que le imponga el Secretario de Estado al concedérsela $^{20}$. Pues bien, mediante la Licence and Agreement, el Gobierno autoriza a la BBC para transmitir en las frecuencias asignadas y de acuerdo con las especificaciones técnicas que se incluyan en ese acuerdo. A cambio de dicha licencia, la BBC se compromete a ofrecer una programación que se reciba en todas las Islas Británicas. En la práctica, la Licence and Agreement es el más importante de los documentos constitucionales de la BBC, ya que en ésta se fijan los derechos y las obligaciones de la Corporación así como las normas de programación. También se desarrollan las obligaciones de servicio público de la BBC y su forma de financiación ${ }^{21}$. Además, respecto de la programación, hay que tener en cuenta las disposiciones contenidas en la Communications Act de 2003, y también en las Broadcasting Acts de 1990 y 1996, que en materia de radiodifusión se mantienen en vigor.

b) Personalidad jurídica

La BBC es una corporación pública, que actúa fuera de la estructura de la Administración pública británica, y que cuenta con un alto grado de independencia. En este sentido, la BBC actúa en nombre propio, es titular de derechos y obligaciones, y tiene capacidad para realizar actos jurídicos ${ }^{22}$.

Por otro lado, es importante resaltar que la actual Royal Charter recoge por primera vez el reconocimiento expreso de la independencia de la BBC. Así se establece en su artículo 6, que dispone que la BBC es independiente respecto de todos los asuntos que conciernan a su programación, a los tiempos de emisión y a la gestión de la Compañía ${ }^{23}$.

view.org.uk/publications/pub_home.html; R. WOLDT, «Der Pert des öffentlichen Rundfunks in der digitalen Ära. Neue Royal Charter für die BBC», en Media Perspektiven, 12/2006, p. 598; T. Prosser, «United Kingdom», op. cit., p. 105.

19 La Licence and Agreement actual se aprobó en julio de 2006, pero ha entrado en vigor, al igual que la Royal Charter, el 1 de enero de 2007, estando vigente hasta el 31 de diciembre de 2016.

20 Royal Charter, art. 49. Actualmente, la competencia para conceder esta Licencia le corresponde al Secretario de Estado de Cultura, Medios y Deporte (Secretary of State for Cultura, Media and Sport). Sin embargo, anteriormente, la Licencia era otorgada por el Secretario de Estado de Patrimonio Nacional (National Heritage).

21 T. Prosser, «United Kingdom», op. cit., p. 103; T. GibBons, «La televisión pública en el Reino Unido», op. cit., p. 37.

22 Royal Charter, arts. 1.1 y 47. Sobre la naturaleza jurídica de la BBC y otras autoridades independientes del Reino Unido, vid., M. SAlvador Martínez, «Los quangos (Reino Unido)», en M. SAlvador MARTínez, Autoridades Independientes, Ariel, Barcelona, 2002, pp. 37 a 85.

23 Efectivamente, es la primera vez en la historia de la BBC que en la Royal Charter se hace una mención expresa a la independencia de la BBC. Sin embargo, la Licence and Agreement de 1996 ya incorporó el re- 
B) Funciones y competencias

a) Funciones de la BBC

La BBC tiene encomendada la prestación del servicio público de radiotelevisión, que desempeña a través de los denominados $B B C$ ' Services, que constan de dos canales de televisión por ondas terrestres ( $B B C$ 1 y $B B C$ 2), dos canales temáticos dentro de la plataforma digital Sky Digital (BBC News y BBC Parliament), cuatro canales digitales ( $B B C$ 3, $B B C 4$, $C B B C$ Channel y CBeebies), y una amplia red de emisoras de radio local y nacional, así como la programación regional y los servicios on line ${ }^{24}$.

Además, la BBC también emite en el extranjero, a través del BBC World Services, que proporciona, en más de treinta lenguas diferentes, información a través de distintos canales de televisión digital o vía satélite, radio y diferentes servicios on $\operatorname{line}^{25}$.

Finalmente, y aparte de la prestación del servicio público de radio y televisión, desde 1996 se permite a la BBC ofrecer servicios comerciales, siempre que cuenten con la aprobación del Secretario de Estado ${ }^{26}$.

Las funciones de la BBC, definidas en la Royal Charter en términos muy amplios y generales ${ }^{27}$, pueden clasificarse en tres categorías: prestaciones de servicio público, servicios de valor añadido y servicios comerciales.

a) Las prestaciones de servicio público: El primer objetivo de la BBC es proporcionar el servicio público de la radiotelevisión, ya sea por medios analógicos o digitales, emitiendo programas que informan, educan y entretienen, para todo el territorio del Reino Unido ( $B B C^{\prime}$ Services) y para otros territorios de la Commonwealth (World Services) ${ }^{28}$.

Pues bien, en relación con las prestaciones del servicio público de radiotelevisión, la $\mathrm{BBC}$ se rige por las disposiciones establecidas en distintos textos normativos: en primer lugar, por la Communications Act de 2003, que indica las obligaciones de servicio público que han de cumplir todos los operadores de radio y televisión, tanto públicos como privados; en

conocimiento expreso de la independencia de la BBC respecto del Gobierno (Cláusula 2.1 de la Licence and Agreement de 1996 y cláusula 4 de la Licence and Agreement actual).

24 Sobre los distintos canales de radio y televisión que se encargan de la prestación del servicio público de radiotelevisión en la BBC, y que se denominan BBC' Services, vid. Licence and Agreement, cláusula 11.

25 Sobre los servicios de radiotelevisión de la BBC en el extranjero, vid., Licence and Agreement, cláusula 64. BBC Trust, Protocol B4: World Service, de enero de 2007.

26 Los servicios comerciales de la BBC se denominan $B B C$ Worldwide y comprenden actividades tales como la venta de programas, la edición de publicaciones, etc. Sobre los servicios comerciales de la BBC, vid. Licence and Agreement, cláusulas 68 a 74.

27 Royal Charter, art. 5.

28 Royal Charter, arts. 3 a 5. 
segundo lugar, por la Royal Charter y la Licence and Agreement que desarrollan de forma más detallada las previsiones de la Communications Act en relación con las obligaciones de servicio público de la BBC; y finalmente, por las normas internas de la propia BBC, que terminan de concretar y de completar este marco normativo.

En primer lugar, la Communications Act de 2003 establece de forma muy detallada y concreta las prestaciones de servicio público que deben ofrecerse en las cadenas de radio y televisión tanto públicas como privadas; es decir, de acuerdo con esta Ley, tanto las radiotelevisiones públicas como las privadas deben realizar determinadas prestaciones de servicio público, siendo más intensas estas obligaciones para las radiotelevisiones públicas que para las compañías privadas de televisión ${ }^{29}$. En este sentido, dispone que las obligaciones de servicio público de radiotelevisión en el Reino Unido son, en primer lugar, asignar servicios de televisión que aseguren que los programas cubren un amplio abanico de contenidos y que son accesibles para la mayor parte de la audiencia; en segundo lugar, realizar y ofrecer una programación equilibrada y adecuada que satisfaga las necesidades y los intereses de la audiencia; y, en tercer lugar, emitir programas que cumplan con altos estándares de calidad, tanto en su elaboración, como en su contenido, de modo que se garantice la exactitud y la imparcialidad editorial ${ }^{30}$. En segundo lugar, junto con la regulación genérica de la Communications Act de 2003 que, como hemos visto exige determinadas prestaciones de servicio público para todos los operadores de radiotelevisión, tanto públicos como privados, las normas que configuran el estatuto propio de la BBC - esto es, la Royal Charter y la Licence and Agreement - también establecen las obligaciones que les corresponde prestar a la Corporación. En este sentido, la Royal Charter incorpora una serie de obligaciones de servicio público denominadas literalmente «public purposes», que la BBC debe cumplir en el desarrollo de su función, y que constituyen el objetivo principal de la Compañía ${ }^{31}$.

29 Communications Act 2003, de 17 de julio de 2003, secciones 263 a 347.

30 Para cumplir con estas obligaciones de servicio público, de acuerdo con la Communications Act se deben asegurar los siguientes principios: proporcionar información, educación y entretenimiento; proporcionar a los ciudadanos una información amplia, correcta, imparcial y comprensible sobre las noticias y asuntos de actualidad, ocurridos en el Reino Unido o en el extranjero, especialmente, en el caso de noticias o programas de contenido político; ofrecer una amplia cobertura de los acontecimientos deportivos; ofrecer programas sobre temas educativos, de naturaleza educativa o con valores educativos; ofrecer programas relativos a la ciencia, asuntos sociales, materias de importancia internacional, asuntos de especial interés o materias de índole religiosa; prestar una especial atención a la programación destinada a la juventud y la infancia; y, finalmente, ofrecer programas que promuevan y estimulen la diversidad cultural y las tradiciones del Reino Unido. Communications Act 2003, secciones 264 (4) y 264 (6).

31 Las obligaciones de servicio público de la BBC de acuerdo con la Royal Charter son: colaborar con la ciudadanía y la sociedad civil; promover la formación educativa y el aprendizaje; estimular la creatividad y la excelencia cultural; representar al Reino Unido, sus naciones, regiones y comunidades; mostrar el Reino Uni- 
En tercer lugar, estas obligaciones de servicio público recogidas en la Royal Charter han sido ampliadas y desarrolladas por la Licence and Agreement ${ }^{32}$, y también por las normas internas de la BBC aprobadas por el órgano de gobierno de la BBC, el BBC Trust ${ }^{33}$. Así, en la Licence and Agreement se recogen todos los principios y obligaciones de servicio público que deben cumplirse en la programación y en los contenidos emitidos por la BBC, de acuerdo con las previsiones establecidas tanto en la Royal Charter como en la Communications $A c t^{34}$. En virtud de estas normas, la BBC renueva su compromiso de asegurar que los programas mantengan un alto nivel de calidad en todos los aspectos, tanto en la elaboración como en la selección de programas, y de ofrecer un servicio de radiotelevisión correctamente equilibrado que abarque un amplio abanico de $\operatorname{contenidos}^{35}$.

Finalmente, el marco jurídico se completa con un código de conducta de la BBC, denominado literalmente «Principios editoriales» (Editorial Guidelines), que se encarga de fijar un código de buenas prácticas y de establecer los principios a los que debe someterse la preparación y difusión de programas en la $\mathrm{BBC}^{36}$.

b) Los servicios de valor añadido: Los servicios de valor añadido (Ancillary Services) son servicios diferentes a los de radiodifusión y televisión, como, por ejemplo, el servicio de teletexto y otros servicios de tipo interactivo ${ }^{37}$. La prestación de estos servicios de valor añadido se somete a la aprobación previa por parte del Secretario de Estado y debe subordinarse al cumplimiento del objetivo principal de prestación del servicio público de radiodifusión ${ }^{38}$.

do al mundo y el mundo al Reino Unido; y promover otros objetivos, colaborando en el desarrollo para el interés público de las nuevas tecnologías de la comunicación y de los nuevos servicios emergentes, y en especial, liderando el proceso del apagón digital. Royal Charter, arts. 4 y 5. T. Prosser, «United Kingdom», op. cit., p. 104 .

32 Licence and Agreement, cláusulas 5 a 10.

33 El Trust aprobó en enero de 2007 un documento en el que se fijaba el marco de actuación en relación al cumplimiento de estos objetivos de servicio público — BBC Public Purpose Remits: Operating Framework- Posteriormente, en diciembre de 2007, el Trust aprobó otros seis documentos adicionales, cada uno de los cuales se encarga de detallar las obligaciones de servicio de público fijadas en la Royal Charter y que han sido revisados en 2013. Para acceder a estos documentos, vid. http://www.bbc.co.uk/bbctrust/framework/purpose_remits.html

34 Licence and Agreement, cláusulas 43 a 63.

35 T. Gibbons, «La televisión pública en el Reino Unido», op. cit., p. 39.

36 Las Editorial Guidelines, también conocidas como, Producer's Guidelines, fueron aprobadas el 29 de junio de 2005 y establecen los valores y principios que deben tenerse en cuenta en el tratamiento de las noticias, como, por ejemplo, la imparcialidad, la exactitud, la privacidad, la programación política, el correcto tratamiento de las minorías. Para acceder al documento actualizado, vid. http://www.bbc.co.uk/guidelines/ editorialguidelines/assets/guidelinedocs/Producersguidelines.pdf. Vid, también BBC Trust, Protocol B2: Editorial Standards, de abril de 2014.

37 Royal Charter de 1996, art. 3.(b). N. Reville, «The BBC in the new broadcasting age», en N. Reville, Broadcasting. Law and Practice, Butterworths, London, 1997, p. 5. Department of National Heritage, The Future of the BBC, Serving the Nation Competing Worldwide, 1994.

38 Royal Charter, art. 5.2. 
c) Los servicios comerciales: Los servicios comerciales (Commercial Services), de acuerdo con la definición dada por la Royal Charter de 1996, tienen por objeto proporcionar servicios de radiodifusión, por medios analógicos o digitales, mediante programas de información, educación y entretenimiento financiados por publicidad, suscripción, sponsoring, sistema de pago por visión, o cualquier otro medio de financiación privada - ya fuera de recepción en abierto para el público en general y de forma gratuita, ya fuera disponible únicamente bajo demanda individual, en codificado o no, en el Reino Unido o en el extranjero ${ }^{39}$.

La prestación de servicios comerciales por parte de la BBC, como algo complementario a la prestación del servicio público de radiotelevisión, se reconoció por primera vez en la Royal Charter de $1996^{40}$ y ha sido desarrollada en la Royal Charter actual. En este sentido, se dispone que la BBC podrá realizar servicios comerciales, de forma subsidiaria, y siempre que éstos sean compatibles con sus obligaciones de servicio público. Además, esos servicios comerciales deberán establecerse en un Acuerdo Marco autorizado por el Secretario de Estado ${ }^{41}$.

Esta restricción a la prestación de servicios comerciales se recoge también en la Licence and Agreement, que dispone que la BBC sólo podrá ofrecer servicios comerciales de forma complementaria a su función principal de prestación de servicio público de radiotelevisión y prohíbe que la BBC proporcione directamente servicios comerciales, como había hecho hasta ahora, es decir, la BBC sólo podrá prestar servicios comerciales de manera indirecta, mediante acuerdos con otras empresas o entes ${ }^{42}$.

\section{b) Competencias}

Como ya hemos visto, la función de la BBC es la prestación del servicio público. Por ello, de acuerdo con la Royal Charter, el objetivo principal de la BBC consiste en la promoción de sus «objetivos públicos», literalmente public purposes. Para conseguir esos objetivos, la BBC dispone de amplias competencias regula-

39 M. Cabe/R. Collins/P. Crowther, «Regulating the BBC» en Telecommunications Policy 28 (2004), pp. 256 y 257.

40 Royal Charter de 1996, art. 3.c).

41 Royal Charter, arts. 3.3 y 5.2 .

42 En este sentido, se establece que la participación de la BBC en servicios comerciales únicamente será válida si cumple con los siguientes criterios: $1 .^{\circ}$ Que los servicios comerciales sean compatibles con sus obligaciones de servicio público; 2. ${ }^{\circ}$ Que se acredite su eficiencia comercial. 3..$^{\circ}$ Que no pongan en peligro la buena reputación de la BBC o el valor de la marca de la BBC. 4. ${ }^{\circ}$ Que cumplan con las normas de defensa de la competencia y, en particular, que no perjudiquen ni distorsionen el mercado. Licence and Agreement, cláusulas 68 y 69. Sobre los servicios comerciales, vid. también BBC Trust, Protocol B1: Strategic and financial approvals, de abril de 2014 y BBC Trust, Protocol C4: Commercial Services, de abril de 2015. 
doras, en las que concreta las obligaciones de servicio público. Por otro lado, la Royal Charter dispone que, de forma complementaria la BBC está facultada para mantener, establecer o adquirir filiales, eso sí, siempre que estén relacionadas con su actividad radiotelevisiva y con sus objetivos públicos ${ }^{43}$.

La BBC está facultada para gestionar la producción y emisión de programas, realizar transacciones comerciales, adquirir propiedades, editar publicaciones, mantener centros de documentación, buscar noticias por todo el mundo, y organizar y financiar conciertos y otras formas de entretenimiento relacionadas con su actividad radiotelevisiva ${ }^{44}$.

Además, hay que resaltar que la BBC tiene potestad reglamentaria, es decir, tiene capacidad normativa para dictar disposiciones relativas a su programación o a los principios que han de inspirar su actividad ${ }^{45}$.

\section{c) Autonomía funcional}

Una de las características que definen al servicio público de la radiotelevisión británica es su independencia respecto del Gobierno. En este sentido, y por primera vez en la historia de la BBC, la Royal Charter recoge expresamente el principio de la independencia de la BBC respecto del Gobierno. Hay que señalar, no obstante, que la Licence and Agreement de 1996 ya recogía en su texto una mención expresa a la independencia de la BBC, pero en esta ocasión, con la redacción de la nueva Royal Charter, se decidió también que se incluyera en la Carta la referencia a la independencia, con el fin de darle una mayor relevancia jurídica ${ }^{46}$.

No obstante, y aunque constituye un hecho relevante el reconocimiento expreso de la independencia en las normas que conforman el estatuto jurídico de la BBC, llama la atención que, a pesar de ello, ninguna de estas normas jurídicas prevén ningún tipo de mecanismo de garantía que pueda utilizar, en su caso, la BBC para defenderse de cualquier ataque a su independencia por parte de los poderes públicos, en especial, del Gobierno. Es más, las normas reguladoras de la $\mathrm{BBC}$ ponen de manifiesto que la relación con el Gobierno es, al menos, formalmente, de subordinación.

En este sentido, en la Communications Act se conceden al Gobierno extensos poderes para intervenir en la televisión, tanto pública como privada ${ }^{47}$. Del mismo modo, las normas que configuran el estatuto jurídico de la BBC (Royal Charter y Licence and Agreement) también contemplan esta posibilidad. Sin embargo, y a

43 Royal Charter, art. 3.3.

44 T. GibBons, «La televisión pública en el Reino Unido», op. cit., p. 37.

45 Así, por ejemplo, podemos citar las Editorial Guidelines, que se asemeja a un código ético o de conducta; BBC Trust, Protocol B2: Editorial Standards, de abril de 2014.

46 Royal Charter, art. 6.

47 Royal Charter, art. 54; Licence and Agreement, cláusula 81. 
pesar de que formalmente el Gobierno está facultado para influir o intervenir en el funcionamiento y en los contenidos de la $\mathrm{BBC}$ o de las radios y televisiones privadas, en la práctica, en muy pocas ocasiones el Gobierno ha hecho uso de estas facultades de control. La razón principal, en opinión de la doctrina, es que existe un «pacto no escrito» entre los grupos políticos y los gestores de la televisión en virtud del cual se asegura la independencia de la BBC a cambio de limitar la información a lo que resulta políticamente aceptable, o, «políticamente correcto». Esto es lo que denominan "política de acomodación» ${ }^{48}$. De acuerdo con este pacto, la BBC se identifica con los intereses de la nación, es decir, de la BBC se espera que en tiempos de crisis se adhiera a los objetivos nacionales ${ }^{49}$.

Por otro lado, este pacto de defensa del interés nacional hace a la Corporación especialmente vulnerable a las críticas por su manera de tratar las noticias y los asuntos de actualidad, especialmente cuando esa información discrepa con el Gobierno $^{50}$.

\section{C) Organización interna}

Por lo que se refiere a su organización interna, la BBC ha tenido importantes cambios con la entrada en vigor de la Royal Charter de 2006. En la actualidad, la $\mathrm{BBC}$ se organiza en torno a dos órganos principales: el BBC Trust, que viene a sustituir al anterior Board of Governors y el Consejo Ejecutivo (Executive Board), órgano encargado de funciones ejecutivas y de gestión. Por otro lado, se mantiene la figura del Director General, pero con funciones mucho más limitadas, pues la mayor parte de estas funciones las ha asumido ahora el Consejo Ejecutivo. Y, finalmente, los Consejos Nacionales de Radiodifusión y los Consejos Regionales han sido sustituidos por los Audience Councils, que se encargan de desempeñar funciones asesoras ${ }^{51}$.

Con la nueva organización de la BBC establecida en la Royal Charter, el $B B C$ Trust y el Consejo Ejecutivo desempeñan un papel esencial en la organización y funcionamiento de la Compañía, aunque se distinguen claramente las

48 T. GibBons, «La televisión pública en el Reino Unido», op. cit., p. 46.

49 Así ha ocurrido en distintos momentos a lo largo de la historia de la Corporación y, de manera muy especial durante la Segunda Guerra Mundial, en la que la BBC fue abiertamente un altavoz del Gobierno y recurría de manera constante a la autocensura, o más recientemente, con la posición de la BBC abiertamente unionista en relación con la celebración del referéndum de independencia celebrado en Escocia

50 Así ha ocurrido, por ejemplo, en la crisis de Suez, en los años 50, en el tratamiento informativo dado a la «guerra de las Malvinas». (Falklands) en los años 80, en los casos de financiación ilegal del partido conservador en los años 90, o más recientemente en relación con la participación del Reino Unido en la Guerra de Irak, ... casos todos ellos en los que se hizo ostensible una postura realmente independiente de la Corporación. R. Negrine, Politics and the Mass Media in Britain, Taylor \& Francis, London, 1994, pp. 112 a 115; T. Gibbons, «La televisión pública en el Reino Unido», op. cit., p. 49. V. Adams, The Media and the Falklands Campaign, Palgrave, London, 1986.

51 T. Prosser, «United Kingdom», op. cit., p. 105. 
funciones y competencias que desarrollan cada uno de ellos. El Trust se encarga de definir la estrategia general de la BBC así como de ejercer las tareas de dirección y control sobre el Consejo Ejecutivo. En este sentido, el Trust tiene como función principal la de garantizar el interés público y proteger los intereses de la audiencia. Por su parte, el Consejo Ejecutivo se encarga de desarrollar y ejecutar los servicios y funciones de la BBC de acuerdo con las directrices y objetivos fijados por el Trust, así como de realizar las labores ejecutivas y de gestión de la Compañía ${ }^{52}$.

\section{a) El BBC Trust}

El BBC Trust es el órgano central de la BBC, que viene a sustituir al anterior Consejo de Gobernadores, y que tiene atribuidas las principales competencias de la Corporación ${ }^{53}$.

El Trust es el órgano soberano de la BBC y controla las funciones del Consejo Ejecutivo, manteniendo su independencia respecto de este órgano. La Royal Charter establece claramente la posición de supremacía del Trust respecto del Consejo Ejecutivo. Así, en los casos en los que el Consejo Ejecutivo desempeñe tareas que sean de competencia del Trust —ya sean de aprobación, supervisión, revisión o ejecución-, prevalece la decisión del Trust sobre la del Consejo Ejecutivo, sin perjuicio de las funciones de supervisión y control que le corresponden a la $\mathrm{OFCOM}^{54}$. No obstante, y aunque el Trust es el órgano rector de la BBC, tanto este órgano como el Consejo Ejecutivo tienen claramente definidos sus roles y responsabilidades ${ }^{55}$.

a) Composición y sistema de elección de los miembros: El Trust de la BBC está formado por el Presidente, el Vicepresidente y, como mínimo, diez Consejeros (literalmente, Trustees, fideicomisarios), de los cuales cuatro de ellos representan a las diferentes regiones que componen el Reino Unido: uno a Inglaterra, uno a Gales, uno a Escocia y otro a Irlanda del Norte ${ }^{56}$.

52 Royal Charter, arts. 7 y 8. T. White Paper on Charter Review, para. 9.5.2; I. KATSIREA, Public broadcasting and European Union. A comparative examination of public service obligations in six Member States, Kluwer Law International, 2008, p. 126.

53 Por lo que se refiere a la naturaleza jurídica del BBC Trust, la propia Royal Charter aclara que este término (literalmente, «fiduciario») se utiliza para hacer referencia a un órgano encargado de garantizar el interés público y la confianza del público, pero no se usa de forma técnica, y, por lo tanto, no son de aplicación las normas generales sobre fideicomisos. Royal Charter, art. 12.

54 Royal Charter, art. 9.

55 White Paper on Charter Review, paragraph 9.5.2. En la doctrina, T. Prosser, «United Kingdom», op. cit., p. 107. Así, por ejemplo, el Trust responde directamente frente a la audiencia. Este novedoso sistema de control por parte de la audiencia se ha visto reforzado por una obligación sin precedentes de publicidad y transparencia en todas las actividades del Trust.

56 Además, para poder ser elegido miembro del BBC Trust en representación de una nación del Reino Unido, la Royal Charter establece algunos requisitos adicionales, como, por ejemplo, que demuestren conoci- 
Como se puede apreciar, la composición del Trust es idéntica a la del anterior Consejo de Gobernadores. Sin embargo, las mayores diferencias se encuentran en el sistema de elección de los miembros que componen el Trust. Así, en el sistema anterior el nombramiento de los Governors era decidido por el Gobierno, y el procedimiento de selección no era público; ni siquiera se facilitaba al Comité de Selección de Patrimonio Nacional (órgano administrativo encargado formalmente de realizar la selección previa de los Gobernadores) los nombres de los candidatos a Gobernador de la $\mathrm{BBC}^{57}$.

En la actualidad, los miembros del Trust son nombrados por la Reina en Consejo, siguiendo las indicaciones del Primer Ministro, lo que supone, en la práctica, que la función de la Reina es meramente formal, y el nombramiento de los Trustees se decide en el Gobierno ${ }^{58}$. Ahora bien, actualmente, el proceso de selección de los miembros del Trust se desarrolla de forma través de la Office of the Commissioner for Public Appointments mediante un determinado procedimiento que se conoce como «los principios Nolan» ${ }^{59}$. Con ello se ha intentado cambiar la imagen de clientelismo político que tenía el órgano rector de la Corporación.

El mandato de los Trustees tiene una duración de 5 años, si bien puede ser reelegido por otro mandato más, con la misma duración ${ }^{60}$.

En cuanto su funcionamiento, hay que indicar que éste se rige por las normas internas aprobadas por el propio Trust, para reforzar su independencia y como consecuencia de su capacidad de autorregulación ${ }^{61}$.

El Trust puede actuar en pleno o en comisiones ${ }^{62}$ y sus reuniones están presididas por el Presidente o, en su ausencia, por el Vicepresidente, y se

mientos de la cultura, tradiciones y problemas de la región a la que van a representar. Por otro lado, y aparte de la función que desempeñan como miembros del Trust, son también quienes presiden los Audience Councils de cada una de estas regiones. Royal Charter, art. 14.

57 Eso hizo que desde los años 80 fuera notorio que los miembros del Consejo de Gobernadores eran personas que simpatizaban con la política del Gobierno. Sobre esta cuestión vid. M. SALVAdOr MarTíNEZ, Autoridades Independientes, op. cit., p. 40.

58 T. Gibbons, «La televisión pública en el Reino Unido», op. cit., p. 36.

59 En 1995 se constituyó un Comité, el Comité Nolan, encargado de reformar el sistema de elección de los quangos del Reino Unido. Este comité proponía establecer criterios públicos claros para la elección de los miembros de estos organismos así como reforzar los mecanismos de control externo de los nombramientos. En desarrollo de estas propuestas, se creó en noviembre de 1995 el Commissioner for Public Appointments, cuya primera tarea fue publicar un Code of Practice for Public Appointment Procedures.

60 Por lo que se refiere a su cese, un miembro del Trust puede cesar por alguna de las siguientes causas: finalización del mandato; por renuncia, mediante escrito dirigido al Secretario de Estado; por cese acordado por el Gobierno; si es declarado insolvente, sus bienes han sido embargados, está en concurso de acreedores o suspensión de pagos; si sufre desórdenes mentales o ingresa en un hospital psiquiátrico o es declarado incapaz por decisión judicial; en caso de ausencia continuada a las reuniones de la Corporación durante más de tres meses sin el consentimiento de la Corporación, y sin causa justificada, que provoque que el Trust considere que ese puesto queda vacante. Royal Charter, arts. 15 y 16.

61 Royal Charter, art. 19. BBC Trust Standing Orders, de 1 de abril de 2014.

62 El Trust puede crear comisiones formadas por los Trustees, que tendrán las mismas funciones y poderes que el propio pleno, aunque deben establecerse claramente la manera en la que estas comisiones infor- 
celebran generalmente en Inglaterra, aunque también pueden celebrarse en Escocia, Gales e Irlanda del Norte, con la periodicidad que decida el Trust $^{63}$. Para que exista quórum es necesario que estén presentes, al menos, cuatro miembros del Trust. Y los asuntos se deciden por mayoría de votos de los miembros presentes en la reunión y, en caso de empate, el Presidente tiene voto de calidad ${ }^{64}$.

En relación con el estatuto personal de los miembros del BBC Trust, la $\mathrm{BBC}$ ha acordado un Código de Conducta en el que se establece la posición jurídica de los miembros del Trust, así como los principios a los que debe ajustarse en el desarrollo de su actividad: régimen de incompatibilidades, aceptación de obsequios, viajes, dietas, actividades políticas o comentarios públicos en relación a temas relacionados con la BBC, etc.

b) Competencias: Como hay hemos indicado, el Trust es el órgano rector de la BBC y tiene atribuida la función de definir los objetivos y estrategias de la Corporación, y controlar la actividad que ésta lleva a cabo, mientras que las funciones ejecutivas y de gestión corresponden al Consejo Ejecutivo y al Director General ${ }^{65}$.

De acuerdo con la Royal Charter, el Trust tiene como función principal garantizar el interés público y proteger los intereses de la audiencia así como asegurar el buen uso de los ingresos por el canon ${ }^{66}$. Y para cumplir con este objetivo, el Trust ejerce determinadas funciones, tanto genéricas como específicas.

Entre las funciones genéricas destacan las siguientes: establecer la dirección estratégica general de la BBC dentro del marco fijado en la Royal Charter y en la Licence and Agreement; la gestión de los ingresos por el canon y otros recursos; aprobar los presupuestos y diseñar la estrategia financiera de la Corporación; el desarrollo del interés público en la BBC, especialmente los intereses de los contribuyentes, es decir, de los que pagan el canon; asegurar el cumplimiento efectivo de los objetivos públicos; y finalmente, supervisar la actuación del Consejo Ejecutivo en el desempeño de sus funciones ${ }^{67}$.

Por su parte, las funciones específicas consisten en: primero, definir y aprobar los criterios de actuación y objetivos de la BBC, planificar los servicios, programas y actividades de la Corporación, así como controlar hasta qué

man al Trust de las funciones llevadas a cabo. Asimismo, el Trust desarrollará un examen o revisión anual de las funciones delegadas a las distintas comisiones para asegurar que la delegación es adecuada y que se está llevando a cabo de forma diligente. Royal Charter, art. 20 y BBC Trust Standing Orders, art. 17.

63 BBC Trust Standing Orders, arts. 7 y 8.

64 BBC Trust Standings Orders, arts. 3 a 6.

65 Royal Charter, art. 7.

66 Royal Charter, art. 22.

67 Royal Charter, art. 22. 
punto se han logrado estos objetivos y se reflejan las necesidades y los intereses del público; segundo, defender los intereses de la audiencia y los contribuyentes. Para ello, es el encargado de establecer el marco para la tramitación de quejas y reclamaciones, además de asegurar que cualquier comentario, propuesta o queja son debidamente atendidos por la Corporación; tercero, asegurar y garantizar la independencia de la BBC; cuarto, controlar y supervisar el cumplimiento de las obligaciones legales y contractuales de la Corporación de acuerdo con los más altos estándares de transparencia, corrección, exactitud e imparcialidad; quinto, ejercer una gestión rigurosa de la financiación del servicio público, mediante la puesta en marcha de controles financieros, además de asegurar que los procedimientos para la recaudación del canon son eficientes, apropiados y proporcionales; sexto, establecer y aprobar los acuerdos marco de los nuevos servicios de la BBC, con independencia de que se requiera el consentimiento del Secretario de Estado; séptimo, nombrar al Director General de la Corporación, que ocupará y cesará en su cargo de acuerdo con los términos establecidos en su nombramiento; octavo, el Trust también tiene potestad reglamentaria, ya que tiene capacidad para aprobar normas internas sobre cuestiones de funcionamiento interno y otros asuntos más específicos. Estas normas internas se denominan protocolos ${ }^{68}$. Por último, ejercerá funciones ejecutivas en relación con el Trust unit y tendrá todas las funciones que, bien de forma expresa, bien de forma tácita, le sean conferidas en la Royal Charter o en la Licence and Agreement ${ }^{69}$.

\section{b) El Consejo Ejecutivo (Executive Board)}

El Consejo Ejecutivo (Executive Board) se encarga de llevar a cabo funciones ejecutivas y de gestión, siempre bajo la dirección, el control y la supervisión del Trust.

a) Composición y sistema de elección: El Consejo Ejecutivo consta de miembros ejecutivos y no ejecutivos. El número de miembros del Consejo Ejecutivo se decide por el propio Consejo, con la aprobación del Trust. Ahora bien, deben cumplirse dos reglas: la primera, es que siempre ha de

68 Royal Charter, arts. 25 a 27. Entre los protocolos que pueden aprobarse, la Royal Charter enumera alguno de ellos, como el protocolo en el que se fijan el funcionamiento interno del Trust; el protocolo en el que se concretan las relaciones entre el Consejo Ejecutivo y el Trust y el reparto de tareas entre ambos órganos; el protocolo en el que se detalle la manera en la que se van a hacer cumplir las opiniones de la audiencia en lo relativo a la programación y a los objetivos de la Corporación; o el Protocolo en el que se han de fijar los estándares de apertura y transparencia. T. Prosser, «United Kingdom», pp. 107 y 108.

69 Royal Charter, art. 24. 
haber como mínimo cuatro miembros no ejecutivos, y la segunda, es que además el total de consejeros no ejecutivos no puede ser inferior a un tercio ni superior a la mitad del total de miembros que componen el Consejo Ejecutivo $^{70}$. En la actualidad el Consejo Ejecutivo está formado por 10 consejeros ejecutivos y 6 consejeros no ejecutivos.

Todos los miembros del Consejo Ejecutivo son nombrados por el propio Consejo, a propuesta de la Comisión de nombramientos ${ }^{71}$, excepto en el caso del nombramiento del Presidente, que es designado por el Trust y que, además, en la práctica, ocupa el puesto de Director General de la Corporación ${ }^{72}$. En la propuesta de nombramiento no sólo se incluye el nombre de la persona propuesta para ocupar ese puesto, sino también los términos de su mandato. Las funciones del Presidente del Consejo Ejecutivo se limitan a dirigir las reuniones del Consejo, y tiene voto de calidad en caso de empate ${ }^{73}$.

b) Duración del mandato: El mandato de los miembros del Consejo Ejecutivo se determina en el momento de su nombramiento, aunque, en principio tiene la misma duración que la de los miembros del Trust, es decir, cinco años ${ }^{74}$.

Por lo que se refiere a las causas de cese de los miembros de Consejo Ejecutivo, aparte de la renuncia voluntaria por parte del Consejero, la Royal Charter prevé que el cese puede ser acordado por el propio Consejo Ejecutivo, a propuesta del Presidente del Consejo, en el caso de miembros no ejecutivos, y por el Trust, en el caso de cese de miembros ejecutivos ${ }^{75}$.

c) Funcionamiento: Por lo que se refiere a su funcionamiento, al igual que ocurre con el Trust, éste se rige por las normas internas del Consejo, en las que se detalla la convocatoria de reuniones, el quórum, las mayorías necesarias para adoptar acuerdos, etc, además de por una norma de carácter re-

70 Royal Charter, art. 28.

71 Para el nombramiento de los miembros del Consejo ejecutivo, existen dos comisiones distintas de selección, en función del tipo de consejero (ejecutivo-no ejecutivo). Así, en el caso del nombramiento de los consejeros ejecutivos el comité de selección está formado por cuatro vocales, de los cuales, uno de ellos será el Presidente del Consejo Ejecutivo, otro miembro más ejecutivo, elegido por el Presidente y dos miembros no ejecutivos. En el caso del nombramiento de los consejeros no ejecutivos, el comité de selección está formado también por cuatro miembros, dos ejecutivos y no ejecutivos, pero en este caso, el comité será presidido por un consejero no ejecutivo. Además, en este caso, el nombramiento de los consejeros no ejecutivos ha de ir ratificado por el Trust. Vid. artículos 30 y 31 de la Royal Charter. Vid., también, BBC Trust, Protocol F1: Appointments to and remuneration of the Executive Board, de febrero de 2012.

72 Aunque en la Royal Charter se establece una regulación específica para ambos cargos, incluso la posibilidad de que el Director General no ocupe la presidencia del Executive Board, en la práctica, quien ocupa la Dirección General de la BBC ostenta, a su vez, la Presidencia del Consejo Ejecutivo. Royal Charter, arts. 29 y 30 .

73 Royal Charter, arts. 32 y 33. T. Prosser, «United Kingdom», op. cit., p. 107.

74 Royal Charter, art. 32.1 y 33.1.

75 Royal Charter, art. 33. 
glamentario aprobada por el Trust ${ }^{76}$. En principio el Consejo Ejecutivo se reúne una vez al mes como mínimo, y las reuniones se celebrarán, generalmente en Londres, aunque podrán celebrarse en otras localidades del Reino Unido, siempre que lo decida el Consejo ${ }^{77}$. Para que éstas sean válidas, se exige un quórum de 5 miembros ${ }^{78}$. Por lo que se refiere a las decisiones, la mayor parte se adoptarán por mayoría simple, excepto en los casos en los que se exija otra mayoría ${ }^{79}$. Además, en caso de empate, el Presidente - 0 en su ausencia, la persona que presida la reunión - tiene voto de calidad.

d) Funciones: El Executive Board se encarga de las tareas ejecutivas de la BBC, y en concreto de lo siguiente: el desarrollo de los servicios de la $\mathrm{BBC}$ de acuerdo con los principios y las directrices fijados por el Trust; la dirección editorial y de producción creativa de la $\mathrm{BBC}$; la realización de las labores ejecutivas y de gestión de la BBC; asegurar el cumplimiento de las previsiones legales y reglamentarias en el desarrollo de los servicios de la BBC; solicitar al Trust la adopción de determinados acuerdos o decisiones relativas al funcionamiento de la Compañía; realizar las tareas de contabilidad y de gestión financiera de la Compañía; y dirigir las operaciones financieras y comerciales de la $\mathrm{BBC}^{80}$.

\section{c) El Director General}

El Director General de la BBC es el director ejecutivo y el editor jefe de la Corporación ${ }^{81}$. Forma parte del Consejo Ejecutivo como miembro ejecutivo del mismo, e incluso puede presidir este órgano. Es nombrado por el Trust ${ }^{82}$.

Por lo que se refiere a las funciones del Director General, en la Royal Charter únicamente se indica que ostenta el cargo de director ejecutivo y editor jefe de la Corporación. En este sentido, el Director General se ocupa de la línea editorial de la BBC y de la parte creativa, y, en los casos en los que ostenta también el Cargo de Presidente del Consejo Ejecutivo, desempeña todas las tareas inherentes a ese $\operatorname{cargo}^{83}$.

76 Royal Charter, art. 34. Vid. Executive Board Standing Orders.

77 Executive Board Standing Orders, art. 5.

78 Executive Board Standing Orders, arts. 4 y 11.

79 Executive Board Standing Orders, arts. 5 a 12.

80 Royal Charter, art. 38. T. Prosser, «United Kingdom», op. cit., p. 107. Sobre la dirección de los servicios comerciales por parte del Consejo Ejecutivo, vid. BBC Trust, Protocol B1: Strategic and financial approvals, de abril de 2014.

81 Royal Charter, art. 40.

82 BBC Trust, Protocol A1: Appointments to and remuneration of the Executive Board, art. 2.

83 Royal Charter, art. 40.2. En la actualidad, las funciones y el poder que ostenta el Director General dentro de la organización de la BBC es mucho más reducido que el que ha tenido a lo largo de la historia. Recordemos que hasta la entrada en vigor de la actual Royal Charter, en enero de 2007, el Director General tenía prácticamente todas las funciones que ahora desempeña el Consejo Ejecutivo. 


\section{d) Los Audience Councils}

Los Audience Councils, que han venido a sustituir a los anteriores Consejos Nacionales de Radiodifusión (National Broadcasting Councils), son órganos consultivos, que tienen por objeto informar al Trust sobre las distintas perspectivas y opiniones de la audiencia para que sean de utilidad en el trabajo del Trust. En la medida en la que representan a distintas comunidades geográficas del Reino Unido, actúan de enlace entre estas comunidades y la BBC, proporcionando al Trust la opinión de la audiencia sobre la manera en la que la Corporación está desarrollando el servicio público ${ }^{84}$. Estos Consejos no tienen mucho poder efectivo, pero sí una considerable influencia derivada del hecho de que la BBC suele considerar seriamente sus propuestas. De hecho, siempre que la BBC decide lanzar un nuevo servicio al mercado, modificarlo o incluso retirarlo, previamente lo somete a la consideración de los Audience Councils ${ }^{85}$.

En la actualidad existen cuatro Audience Councils de la BBC, que se denominan, respectivamente, Audience Council de Inglaterra, Audience Council de Escocia, Audience Council de Gales y Audience Council de Irlanda del Norte ${ }^{86}$. Cada uno de ellos estará presidido por el miembro del Trust que representa a cada una de estas regiones ${ }^{87}$.

e) El personal

La BBC tiene autonomía para contratar el personal que considere necesario para el desarrollo de sus funciones y, además, para fijar los salarios y las condiciones laborales de los mismos ${ }^{88}$. Hay que indicar que a pesar de ser un ente público, el personal de la BBC no pertenece al civil service (a la función pública), sino que es personal contratado ${ }^{89}$. Los trabajadores de la BBC son contratados por el Consejo Ejecutivo, excepto el personal propio del Trust, el denominado Trust Unit, que queda bajo el control ejecutivo de este órgano ${ }^{90}$.

84 Royal Charter, art. 39. En la doctrina, T. MADGE, Beyond the BBC: Broadcasting and the Public in the 1980s, The MacMillan Press, Edinburgh, 1989; T. GibBons, «La televisión pública en el Reino Unido», op. cit., p. 37.

85 BBC Trust's Audience Engagement Protocol, art. 3.2.2.

86 Royal Charter, art. 39.3.

87 Royal Charter, art. 14.

88 Royal Charter, art. 41.

89 M. Salvador Martínez, Autoridades Independientes, op. cit., p. 65. T. Prosser, «United Kingdom», op. cit., p. 105 .

90 Royal Charter, arts. 41 a 44. Sobre la organización y funcionamiento del Trust Unit, vid. BBC Trust, Protocol A2: BBC Trust Unit, de noviembre de 2006. 


\section{D) Fuentes de financiación}

En los primeros tiempos de la BBC, desde su creación, los ingresos de la compañía provenían de dos fuentes distintas: un impuesto sobre las ventas de los aparatos de radio y un canon por el uso de estos aparatos ${ }^{91}$. Sin embargo, este sistema no fue del todo satisfactorio, ya que la situación financiera de la Compañía dependía, en cierta medida, del éxito comercial de los equipos radiofónicos británicos, con lo que sus ingresos se vieron pronto amenazados por los comportamientos de una audiencia que, o bien compraba aparatos extranjeros (que no estaban sujetos al impuesto sobre la venta) o bien los fabricaba en su casa ${ }^{92}$.

A la vista de esta situación, en 1923 se constituyó un Comité de Radiodifusión (Broadcasting Commitee), que recomendó que la compañía no siguiese dependiendo de los ingresos obtenidos por la venta de los aparatos de radio, sino que se financiase exclusivamente por los ingresos provenientes del canon por el uso de la radiotelevisión ${ }^{93}$. Y este sistema es el que se ha venido utilizando desde entonces hasta nuestros días para la financiación del servicio público de radiotelevisión, aunque en la actualidad se ha abierto un intenso debate al respecto, ya que con la irrupción de nuevos dispositivos móviles y audiovisuales, cada vez hay menos hogares con aparatos de televisión, lo que está suponiendo un descenso importante de los ingresos de la BBC y que está provocando problemas de financiación para la Corporación.

Por otro lado, debemos recordar que la BBC tiene como principal función la prestación del servicio público de radiotelevisión, pero también desempeña otras funciones, como la difusión de radio y televisión en el extranjero (a través del BBC World Services) y el desarrollo de determinados servicios comerciales. Pues bien, de acuerdo con las normas reguladoras de la BBC, se exige que la BBC establezca un sistema de financiación y una contabilidad distinta e independiente para cada uno de los servicios que presta, es decir, una financiación y contabilidad propia para el servicio público de radiotelevisión, otra para la prestación de los servicios comerciales y otra para los servicios en el extranjero (world services) ${ }^{94}$.

Además, en este sentido, se exige que los ingresos obtenidos por el canon deben utilizarse única y exclusivamente para el servicio público de radiodifusión y se prohíbe expresamente que la BBC utilice esos ingresos para financiar cualquier otro servicio - es decir, el canon no se puede utilizar para sufragar los costes generados en el world services, en los servicios comerciales o en cualquier otro servicio que lleve

91 Wireless Telegraphy Act de 1949, s.1 (1).

92 E. Barendt/L. Hitchens, Media Law. Cases and Materials, Longman, Essex, 2000, p. 72.

93 T. GibBons, «La televisión pública en el Reino Unido», op. cit., p. 36.

94 Licence and Agreement, cláusula 76. Esta diferenciación de servicios y formas de financiación trae causa del Protocolo sobre el sistema de radiodifusión pública de los Estados miembros, anejo al Tratado Constitutivo de la Comunidad Europea, que obliga a todos los Estados miembros que tienen sistema de radiodifusión pública a distinguir claramente entre los distintos servicios que prestan y las formas de financiación de cada uno de ellos. 
a cabo la BBC a solicitud o petición de cualquier departamento del Gobierno" ${ }^{95}$, pues cada uno de estos servicios cuenta con sus propias vías de ingresos.

a) La financiación del servicio público

La financiación del servicio público de radiotelevisión se obtiene únicamente del canon que pagan los ciudadanos que poseen un aparato de televisión en el Reino Unido. Además, se prohíbe expresamente obtener ingresos por publicidad o patrocinio, salvo que exista una autorización del Secretario de Estado ${ }^{96}$.

Como ya hemos visto, desde la creación de la BBC en los años 20, la principal fuente de financiación, en ocasiones, la única, ha sido a través del canon por el uso de los aparatos de radio, primero, y posteriormente, también de televisión. Sin embargo, y a pesar de que la financiación a través del canon es un sistema totalmente asentado en la sociedad británica, el sistema no ha sido del todo satisfactorio, y a lo largo de la historia de la BBC se han ido sucediendo distintas críticas y problemas de aplicación ${ }^{97}$.

En respuesta a estos problemas en los años 80 se constituyó un Comité, denominado comité Peacock, con el objetivo de analizar y, encontrar en su caso, un sistema alternativo para financiar a la BBC, a la vista de las críticas que se hacían a la racionalidad y viabilidad del sistema del canon. Entre las distintas alternativas que se barajaron en el Comité estaba, por ejemplo, la posibilidad de que la BBC se financiara con fondos públicos, mediante una partida presupuestaria incluida en los presupuestos generales del Estado. Sin embargo, esta opción fue rechazada por el Comité, al considerarla constitucionalmente desaconsejable, pues afectaría a la independencia de la $\mathrm{BBC}^{98}$. Otra alternativa que se barajó fue la posibilidad de financiarse con publicidad, pero, al igual que ocurrió el 1923, cuando el Comité de radiodifusión se planteó también esta posibilidad, fue desestimada, al entender que podía afectar negativamente a la calidad de la programación ${ }^{99}$.

95 Este caso es muy excepcional. Por ello, cuando un departamento del Gobierno solicita la prestación de un determinado servicio o la difusión de un determinado programa o información, el Secretario de Estado correspondiente habrá de abonar a la $\mathrm{BBC}$ los gastos ocasionados.

96 Licence and Agreement, cláusula 75.1.

97 Una de las principales críticas es que, en un principio la tasa que se pagaba en concepto de canon era una cantidad fija, por lo que debido al efecto de la inflación, hacía de esta cantidad una fuente de ingresos poco segura, y obligaba a la BBC a solicitar al Gobierno, a intervalos cada vez más cortos, que se incrementase la cuantía del canon para poder subsistir. La segunda de las críticas principales respecto del canon es el carácter regresivo de este impuesto, ya que se debe pagar con independencia de si la televisión se ve poco o mucho y con independencia de los ingresos que tenga cada usuario.

98 En este sentido, el Comité entendía que si la BBC se financiaba con cargo a los presupuestos generales del Estado, la BBC quedaría expuesta al control y al escrutinio por parte del Parlamento, no sólo sobre su gestión, sino posiblemente también sobre su programación, con el riesgo de que pudiera ser sometida a presiones indebidas.

99 T. Gibbons, «La televisión pública en el Reino Unido», op. cit., p. 40. 
Finalmente, el Comité concluyó sus trabajos sin encontrar un sistema de financiación mejor que el canon, por lo que recomendó mantener dicho sistema de financiación, al considerar que, de este modo, se permitía a la BBC perseguir sus objetivos sin verse entorpecida por consideraciones comerciales, que podrían provocar un descenso en la calidad de sus programas, y sin verse sometida a presiones que pudieran afectar a su independencia ${ }^{100}$. Eso sí, para evitar los problemas de financiación que había sufrido la BBC, el Comité recomendó que el canon se fuera actualizando de acuerdo con la inflación. De este modo, se dispondría de una fuente permanente de recursos sin necesidad de renegociación periódica de la cuantía del mismo ${ }^{101}$. Tras estos trabajos, el Gobierno hizo suya la propuesta del Comité Peacock y anunció que iba a actualizar el canon al menos según el IPC, con el fin de incentivar a la BBC a obtener ingresos por otras vías, por ejemplo, a través de suscripciones para la recepción de determinados programas o servicios ${ }^{102}$.

A partir de ese momento la $\mathrm{BBC}$ ha tenido que ir reorganizando de forma constante su sistema de gestión financiera, con el fin de ir reduciendo sus costes y de lograr una adecuada relación coste-eficacia ${ }^{103}$.

Por otro lado, cada vez que se aproxima el momento de renovar una nueva Royal Charter (el marco regulador de la BBC) vuelve a discutirse la idoneidad del canon como sistema de financiación. En este sentido, en los años previos a la aprobación de la actual Royal Charter se produjo un intenso debate sobre esta cuestión, pero como no se llegó a encontrar una alternativa satisfactoria, se ha decidido posponer este debate hasta 2016, fecha en la que se habrá de aprobarse la siguiente Royal Charter. En ese momento se revisará si el canon sigue siendo la forma adecuada de financiar el servicio público ${ }^{104}$.

La cantidad que deben abonar los poseedores de un aparato de televisión en concepto de canon lo fija el Gobierno, en concreto, el Secretario de Estado del Departamento de Cultura, Medios y Deporte. El canon se fija por un periodo de

100 Report of the Peacock Committee on Financing the BBC, Cmnd 9824 (1986). En la doctrina, D. AtKInson, «Public service television in the age of competition», op. cit., p. 56; T. GiBbONs, «la televisión pública en el Reino Unido», op. cit., pp. 40 y 41; E. BAREndt/L. Hitchens, Media Law. Cases and Materials, op. cit., p. 69; N. GARNHAM, «The broadcasting market and the future of the BBC», op. cit., pp. 12 y 13.

101 El Comité tomó el IPC como indicador para actualizar la cuantía del canon, aun a sabiendas de que los costes de la televisión son generalmente más altos, pero su objetivo era estimular a la BBC a una mayor eficiencia en la administración de sus recursos.

102 Home Office, Broadcasting in the '90s: Competition Choice and Quality, 1998; Department of National Heritage, The future of the BBC: serving the Nation, competing Worldwide, 1994, cm.2621 (White Paper). National Heritage Committee, The future of the BBC, 1993-1994, H.C. 77.

103 J. TusA, «Implications of recent changes at the BBC», en Political Quarterly 65 (1994), pp. 6 a 10; M. Cabe/R. Collins/P. Crowther, «Regulating the BBC», op. cit., p. 259 y ss.

104 Uno de los puntos más controvertidos en relación con el canon ha sido si éste puede utilizarse para financiar nuevos servicios de la BBC que pueden no ser accesibles para todos los espectadores y usuarios. Tras múltiples discusiones, se decidió que esos servicios no podrán financiarse por el canon, sino que habrán de sufragarse mediante suscripciones. T. Prosser, «United Kingdom», op. cit., p. 106. 
varios años y va incrementándose de acuerdo con el IPC. En la actualidad, esa cantidad que se cobra a cada propietario de un aparato de radiotelevisión es de 135,50 libras británicas (o 192,921 euros por año).

b) La financiación del World Service

La programación y las emisiones de la BBC en el extranjero (BBC World Services) son financiadas de manera independiente por el Ministerio de Asuntos Exteriores, el Foreign Office $e^{105}$. En este sentido, la secretaría de Estado de Asuntos Exteriores, a través de la Foreign and Commowealth Office (FCO), abona cada año una cantidad a la BBC proveniente de los presupuestos generales del Estado para la financiación de los world services ${ }^{106}$.

c) La financiación de los servicios comerciales

Como ya hemos visto, la Royal Charter de 1996 abrió la posibilidad de que la BBC pudiera financiar sus servicios comerciales mediante publicidad, suscripción, sponsoring o patrocinio, sistemas de pago por visión, o cualquier otro método, con independencia de su forma de difusión (en abierto, bajo demanda individual, de forma codificada o no), e incluso de su ámbito territorial de difusión (ya sea en el Reino Unido o en el extranjero). Estas formas de financiación se someten a la aprobación del Secretario de Estado y se prohíbe expresamente la utilización del canon de televisión para financiar los servicios comerciales. Además, actualmente se exige que el servicio público de televisión tenga una financiación y contabilidad separada de los servicios comerciales ${ }^{107}$.

\section{d) Otros ingresos}

Finalmente, la BBC genera otros tipos de ingresos, de cuantía menor, como son los ingresos generados en la Universidad a Distancia, los ingresos generados por cualquier acuerdo de coproducción; la coproducción o co-financiación, con organizaciones no lucrativas, para la promoción y el fomento de lenguas minoritarias, o los ingresos provenientes por la obtención de premios o galardones ${ }^{108}$. La $\mathrm{BBC}$ también obtiene otros ingresos a través de operaciones comerciales, tales

105 T. GibBons, «La televisión púbica en el Reino Unido», op. cit., p. 40.

106 Licence and Agreement, art. 75.6. BBC Trust, Protocol B4: BBC World Service, de enero de 2007, arts. $1.2,5.3$ y 5.6 .

107 Licence and Agreement, cláusulas 76.

108 Licence and Agreement, art. 75. 
como la venta de programas y publicaciones en mercados exteriores, emisoras de radio o compañías de televisión ${ }^{109}$.

\section{E) Controles}

a) Controles internos

El órgano encargado de supervisar y controlar el correcto funcionamiento de la BBC es el BBC Trust. Así, y como hemos indicado previamente, el Trust es el encargado de garantizar que se cumplen con los objetivos de servicio público, de proteger los intereses de la audiencia así como de controlar que los ingresos provenientes del canon se utilizan de manera adecuada.

En este sentido, en los últimos años el papel del Trust como garante del servicio público se ha hecho más explícito. Así, la Royal Charter y la Licence and Agreement ha incorporado un mecanismo denominado «Public Value Test». Este concepto se ha utilizado en otros ámbitos en los que operan servicios públicos, como la educación o la sanidad, y consiste en que cualquier servicio público debe ofrecer un valor añadido público que puede y debe ser medido y evaluado. En el ámbito del servicio público de televisión, el concepto de «Public Value» es opuesto al objetivo de los operadores comerciales de televisión, que consiste en obtener beneficios para sus accionistas o propietarios. La finalidad de la BBC (y tarea primordial del Trust) es la de crear valor público, es decir, tratar a las audiencias no sólo como consumidores, sino también como miembros de una sociedad, de una comunidad, de modo que a través de los diversos programas y servicios, más allá de las funciones de informar, educar y entretener a la audiencia, también coadyuvan a objetivos públicos más amplios ${ }^{110}$.

109 T. GibBons, Regulating the Media, op. cit., p. 180.

110 Royal Charter, arts. 22 a 25; Licence and Agreement, cláusulas 23 a 33. El proceso de «Public Value Test» consiste en un conjunto de evaluaciones cualitativas y cuantitativas para demostrar que cualquier nuevo servicio de la BBC aporta más beneficios que perjuicios para la sociedad. De este modo, el Trust es el encargado de realizar este «Public Value Test» a cada nuevo servicio que se vaya a ofrecer en la BBC. Este test se realiza en dos fases diferentes: la primera evalúa el impacto sobre el mercado del nuevo servicio que se quiere ofrecer. Esta evaluación será realizada por la OFCOM. La segunda fase consiste en realizar un análisis del denominado "public value assesment», es decir, de sus aportaciones a la sociedad. Para realizar esta segunda evaluación se tienen en cuenta cuatro factores: audiencia, calidad, impacto y valor económico. Una vez realizadas estas dos evaluaciones, el Trust decidirá sobre la idoneidad del lanzamiento del nuevo servicio. Además, se establece que cada cinco años como máximo se encargará una encuesta independiente a 10.000 ciudadanos para determinar el valor público aportado por cada servicio. De este modo, con este sistema se pretende que el ciudadano que paga el canon reciba unos servicios que le aporten valor público. Así, por ejemplo, a finales de 2006 se llevó a cabo el primer "public value test» sobre el servicio de contenidos audiovisuales bajo demanda de la BBC, denominado iMPlayer, que permite el acceso para los ciudadanos del Reino Unido de cualquier contenido audiovisual emitido por los canales de distribución tradicionales de la BBC durante una semana desde su difusión. 
Por otro lado, y respecto a la obligación de garantizar los intereses de la audiencia, el Trust, además de resolver las quejas que se puedan plantear en relación con la BBC, elabora y publica informes anuales, dando cuenta a la audiencia de todo lo que se ha realizado durante ese año ${ }^{111}$.

Finalmente, el Trust se encarga de supervisar el estado financiero de la BBC. Para ello, ha de llevar a cabo una gestión rigurosa del canon y de la financiación del servicio público de televisión, además de establecer procedimientos de contabilidad separados para la financiación de los distintos servicios prestados por la Corporación.

b) Controles externos

a) Control del Parlamento: La BBC está obligada a presentar anualmente al Parlamento un informe sobre la consecución de sus objetivos, el cumplimiento de las obligaciones de servicio público y el estado de sus cuentas ${ }^{112}$. Por lo que se refiere al control financiero, hay que indicar que el Parlamento, a través del servicio de auditoría, pueden revisar el dinero pagado a la $\mathrm{BBC}$ en concepto del pago a la $\mathrm{BBC}$ por los world services, pero no tiene capacidad para controlar los ingresos de la $\mathrm{BBC}$ por el canon, ya que esto es competencia exclusiva de la propia Corporación.

b) Control del Gobierno: Como ya hemos indicado anteriormente, la relación de la BBC con el Gobierno es, formalmente, de subordinación. En este sentido, el Gobierno controla la actividad de la BBC a través de los siguientes medios.

El primero de los poderes de control del Gobierno respecto de la BBC, es el hecho de que la Carta se garantice sólo durante un periodo limitado de tiempo (por regla general, durante diez años). Debemos recordar que es el Gobierno el que periódicamente se encarga de renovar la licencia de emisión (Licence and Agreement), además de aprobar la Royal Charter, en la que se configura el estatuto jurídico de la Corporación. Por ello, y aunque el Gobierno ha reconocido siempre la independencia editorial de la BBC, al menos formalmente, lo cierto es que ésta no tiene un status permanente y ello provoca inquietud e incertidumbre en la propia Corporación, sobre todo cuando se acerca la época de renovación de la Royal Charter ${ }^{113}$. Esta situación de inseguridad jurídica en la que se encuentra la BBC cada vez que hay que renovar sus normas de funcionamiento y la licencia de

111 Royal Charter, art. 45.

112 Royal Charter, art. 24.

113 T. GibBons, «La televisión pública en el Reino Unido», op. cit., p. 46. T. Burns, The BBC: Public Institution and Private Word, op. cit., capítulo VI. Un ejemplo muy claro de ello fue el debate suscitado en el seno de la BBC con la elaboración y aprobación de la Royal Charter de 2006 y todas las noticias relacionadas con la elaboración de la próxima Royal Charter. 
emisión ha provocado que un sector de la doctrina defienda la necesidad de fijar bases legales estables para la BBC. De este modo, por un lado, se garantizaría la permanencia de la Corporación y se podrían concretar sus obligaciones de servicio público, y, por otro, el marco legal daría a la BBC la posibilidad de defenderse de las presiones políticas y de resistir a las presiones de carácter financiero ${ }^{114}$.

En segundo lugar, la BBC necesita la autorización del Secretario de Estado para llevar a cabo determinadas actuaciones, como, por ejemplo, el establecimiento de servicios comerciales, etc. ${ }^{115}$.

Por otro lado, existen también otros poderes del Gobierno más específicos, a través de los cuales se admite la posibilidad de que éste pueda dar algún tipo de orden o instrucción a la BBC. Estas órdenes o instrucciones pueden ser de dos tipos: en primer lugar, la posibilidad de prohibir o vetar la difusión de determinados programas o contenidos, y, en segundo lugar, la exigencia de emitir un determinado contenido o una determinada información.

Por lo que se refiere al primer tipo, las normas reguladoras de la BBC permiten que, en ciertas circunstancias, el Gobierno pueda prohibir o vetar la difusión de un programa determinado o de algún contenido en concreto $^{116}$. En este caso, el Secretario de Estado puede exigir a la Corporación para que se abstenga de emitir una determinada información o un determinado programa. Ese requerimiento ha de hacerse por escrito y debe constar si la prohibición de emitir un programa o cierta información es temporal o definitiva. Por su parte, la BBC está obligada a cumplir con los requerimientos del Gobierno, y el único margen de maniobra de que dispone es que tiene la facultad de hacer público el veto opuesto por el Gobierno, es decir, puede anunciar que un determinado programa o una determinada información ha sido suprimido por órdenes o mandato del Gobierno. De este modo, en cierta manera, la BBC puede protestar públicamente ante cualquier tipo de presión que el Gobierno intente ejercer sobre la Corporación ${ }^{117}$. Esta facultad de

114 T. GibBons, «La televisión pública en el Reino Unido», op. cit., p. 38. E. BarendT, «Legal aspects of the BBC Charter renewal», en The Political Quarterly 65 (1994), p. 20.

115 P. Carey, Media Law, 2007, p. 254.

116 Royal Charter, art. 54.2; Licence and Agreement, cláusula 81. Por su parte, la Communications Act de 2003 establece también una previsión muy similar — prácticamente idéntica — para las radiotelevisiones privadas, Communications Act de 2003, sección 336. Ahora bien, en estos casos, el medio de comunicación está facultado para informar a sus espectadores de que la no difusión de un determinado programa o noticia obedece a órdenes gubernamentales.

117 Licence and Agreement, cláusula 81. Cabe resaltar que aunque esta previsión se encuentra en el apartado relativo a situaciones de emergencia o de defensa, lo cierto es que esta facultad no se limita únicamente a la prohibición de emitir determinados programas o contenidos en situaciones de emergencia. 
veto no se ha utilizado para prohibir un programa en concreto, pero sí para vetar o prohibir la difusión de ciertas informaciones ${ }^{118}$.

La segunda facultad específica que tiene el Gobierno en relación con la $\mathrm{BBC}$ consiste en el poder que tiene cualquier miembro del Gobierno de exigir la difusión de un determinado programa o la emisión de cualquier tipo de comunicado o aviso ${ }^{119}$. Esta facultad se circunscribe únicamente a situaciones de emergencia y, con el fin de proteger la independencia de la Corporación, ésta puede discrecionalmente decidir si hace público que el Gobierno le ha instado a difundir esa información o a emitir ese determinado programa. Esta previsión que se recoge en las normas propias de la $\mathrm{BBC}$ también se establece para los medios privados de radio y televisión en la Communications Act. Ahora bien, en esos casos, y al igual que ocurre con la BBC, el medio de comunicación privado está facultado para informar a los espectadores de que la inclusión de un determinado comunicado o noticia obedece a órdenes gubernamentales ${ }^{120}$.

c) Control de la OFCOM: La OFCOM, autoridad independiente que supervisa el sector audiovisual tiene pocas facultades de control sobre la BBC ${ }^{121}$. La mayoría de las competencias de supervisión se encuadran en aspectos relacionados con los contenidos y en el cumplimiento de los estándares de programación. Lo habitual es que la OFCOM y la BBC celebren acuerdos sobre

118 Así, a lo largo de la historia de la BBC tenemos varios ejemplos. La primera ocasión en la que se utilizó este poder se produjo en los años 20. Durante un año aproximadamente —entre 1927 y 1928 - el Gobierno prohibió que en la BBC se emitiera cualquier información sobre asuntos de naturaleza política, industrial o religiosa, con el fin de abortar una convocatoria de huelga general organizada por varias agrupaciones de trabajadores. La segunda ocasión en la que se utilizó esta posibilidad de veto fue en 1972, cuando el Gobierno británico presentó un requerimiento de veto contra un programa de la BBC sobre terrorismo en Irlanda del Norte, pero finalmente desistió y retiró la prohibición ante la amenaza de la BBC de hacer pública la acción del gobierno de prohibir la difusión de ese programa. En los años 80 y, también en relación con la difusión de información sobre el grupo terrorista IRA se produjo, de nuevo, el veto gubernamental. En esta ocasión, el Secretario de Estado prohibió, en 1988, la emisión de declaraciones directas de los miembros de ciertas organizaciones relacionadas con grupos terroristas de Irlanda del Norte. Esta prohibición fue ampliamente rechazada por los gestores de la Corporación. Por ello, y dado que el requerimiento enviado por el Gobierno prohibía la difusión de «declaraciones directas», la BBC lo interpretó de forma literal y textual, de modo que, para evadir el veto del Gobierno, la Corporación decidió emitir esas declaraciones, pero doblando las voces de las personas que aparecían en la información. Este asunto llegó hasta la Cámara de los Lores, que consideró que el Secretario de Estado había hecho un uso razonable de su facultad de control para combatir el terrorismo. (R V. Sec. of State for the Home Department, ex. p. Brind (1991) 1 All ER 720). E incluso, este caso llegó al Tribunal Europeo de Derechos Humanos, que consideró que esta restricción no era desproporcionada. Finalmente, esta prohibición fue retirada. Brind v. Secretary of State for the Home Department, 1991, 1 A11 ER 720. Tampoco la Comisión Europea de Derechos Humanos consideró admissible la demanda de la República de Irlanda contra dicho bando por infracción del Art. 10 CEDH. Caso Purcell v. Ireland, 1991. T. Gibbons, «La television pública en el Reino Unido», op. cit., p. 48. T. Prosser, «United Kingdom», op. cit., p. 109. R. Craufurd Smith, Broadcasting Law and Fundamental Rights, op. cit., p. 170. App. Nx. 18714/91, Brind et al. v. UK, 77-B D\& R 42.

119 Royal Charter, art. 54; Licence and Agreement, cláusula 81.

120 Communications Act 2003, sección 336.

121 Estas obligaciones se encuentran recogidas en el art. 198 de la Communications Act, y en la cláusula 91 de la Licence and Agreement. 
determinados contenidos ${ }^{122}$ o bien se sometan a lo establecido en el Broadcasting Code, reglamento elaborado por la propia OFCOM y que rige tanto para los medios públicos como privados, aunque tanto las obligaciones como el régimen sancionador varía en función de la naturaleza del medio ${ }^{123}$.

d) Control judicial: Teóricamente la BBC está sometida, igual que cualquier otro sujeto público o privado, al control de jueces y tribunales. En la práctica, sin embargo, éstos han desempeñado un papel muy reducido en el ámbito de la radiotelevisión.

Las pocas intervenciones de los tribunales en materia de radiotelevisión se han desarrollado en relación con las radiotelevisiones privadas, por regla general, por cuestiones relacionadas con los contenidos y, hasta ahora, los tribunales han optado por inhibirse en cierto modo y ratificar las decisiones de las autoridades de control ${ }^{124}$. En este sentido, las decisiones judiciales suelen interpretar que si los gestores de la televisión han obrado razonablemente (en el sentido específico de este término en el derecho público), los tribunales no deben interferir ${ }^{125}$.

Sin embargo, la cuestión de si las propias decisiones de la BBC pueden ser revisadas judicialmente no está todavía resuelta por los tribunales. Así, un sector doctrinal considera que las obligaciones derivadas de la Royal Charter no son judicialmente exigibles, sino que únicamente tienen sanciones políticas. Por el contrario, otro sector de la doctrina afirma que aunque las actividades de la $\mathrm{BBC}$ son revisables judicialmente, el principio de razonabilidad concede a la BBC un amplio margen de discrecionalidad, en el que los tribunales no pueden intervenir. Aquí, de nuevo, vuelve a echarse en falta un marco legal estable de la BBC, pues de ese modo, se podrían establecer claramente los estándares y los objetivos de la corporación y podrían ofrecerse una base explícita para exigirle responsabilidad ante los tribunales ${ }^{126}$.

\section{La Welsh Authority}

La Welsh Authority es la televisión pública de la región de Gales y se regula en la Broadcasting Act de 1990 y en Communications Act de $2003^{127}$. Se encarga de

122 Así, la Ley dispone que se celebrarán acuerdos sobre cuestiones relacionadas con noticias y asuntos de actualidad, cuotas para producciones independientes, cuotas para programas en versión original, elaboración de contenidos para las distintas regiones del Reino Unido, programación especial para discapacitados, etc. Licence and Agreement, cláusulas 92 y 93.

123 P. Carey, Media Law, 2007, op. cit., p. 254.

124 T. Gibbons, «La televisión pública en el Reino Unido», op. cit., pp. 50 y 51.

125 Caso McWhirter, Attorney — General ex rel McWhirter v. Independent Broadcasting Authority, 1973, 1 QB 629; Whitehouse v. Independent Broadcasting Authority.

126 T. GibBons, «La televisión pública en el Reino Unido», op. cit., p. 38.

127 Broadcasting Act de 1990, Parte I; Communications Act de 2003, Parte III, capítulo I. 
la prestación del servicio público de televisión en la región de Gales, cumpliendo con los estándares de calidad, y con la obligación de emitir parte de la programación diaria en galés ${ }^{128}$. El órgano de dirección de la Welsh Authority está formado por de un Presidente, y entre cuatro y ocho miembros, elegidos todos ellos por el Secretario de Estado ${ }^{129}$.

La Welsh Authority se financia a través de un porcentaje sobre el canon de televisión, que se entrega anualmente por el Secretario de Estado, y a través de la publicidad $^{130}$. Por otro lado, y en relación con los controles, la Welsh Authority está obligada a presentar ante el Secretario de Estado un informe anual en el que se incluye su presupuesto, informe que será remitido posteriormente al Parlamento, además de someterse al control por parte de la OFCOM ${ }^{131}$.

\section{Channel 4}

Channel 4 se creó en 1980 por la Broadcasting Act de 1980 y comenzó sus emisiones en $1982^{132}$. Se trata de una corporación pública, sin fines de lucro, que tiene como principal objetivo la realización de programas para las minorías, programas innovadores y experimentales, que, por regla general, no suelen emitirse en las cadenas comerciales, además de un amplio contenido de programas educativos y de noticias e información ${ }^{133}$. Esta función de innovación y creatividad forman parte de las señas de identidad de este canal, y así se recoge expresamente en la Communications Act ${ }^{134}$.

En la actualidad, Channel 4 consta de dos canales: Channel 4, gestionado por la Channel 4 Television Corporation, y está sujeto a la supervisión y regulación de la

128 Broadcasting Act de 1990, arts. 57.2 y 59; Communications Act de 2003, art. 204.

129 Antes de proceder al nombramiento, el Secretario de Estado debe acreditar que el candidato no tiene ningún interés económico o de otra índole que pueda perjudicar el desempeño de sus tareas. Además, el candidato debe facilitar al Secretario de Estado, si éste lo requiere, toda la información que éste considere relevante para comprobar que está preparado para desempeñar ese puesto. Schedule $6, \S 2.3$, y 4 . El mandato de los miembros que componen la Welsh Authority tiene una duración de cinco años. Broadcasting Act de 1990, Schedule 6, §3.

130 A partir de 1993, ese porcentaje es del 32\% del total de ingresos por la tasa de televisión. Broadcasting Act de 1990, arts. 60 y 61. Communications Act de 2003, art. 207.

131 Broadcasting Act de 1990, Schedule 6, § 13. D. Kevin, Regional and local television in the United Kingdom, European Audiovisual Observatory, Estrasburgo, 2015, p. 11.

132 Hasta ese momento solo existían tres canales de televisión: BBC 1 y 2 (el servicio público de radiotelevisión); Channel 3 (medios privados): Tras las recomendaciones efectuadas por el Comité Annan, dicho comité consideró adecuado crear un nuevo canal; Vid. AnNAN: Report of the Committee on the Future of Broadcasting, 1977, Cmnd. 6753. E. Barendt/L. Hitchens, Media Law. Cases and Materials, op. cit., p. 94; P. SCANNELL, «Great Britain: Public service broadcasting, from national culture to multiculturalism», en D. ATKINson/M. Raboy, Public Service Broadcasting: the Challenges of the Twenty-first Century, UNESCO Publishing, 1997, p. 92.

133 P. Carey, Media Law, 2007, op. cit., p. 255; T. GibBons, «La televisión pública en el Reino Unido», op. cit., p. 52.

134 Communications Act 2003, sección 265 (3). 
OFCOM $^{135}$, y S4C (Sianel Pedwar Cymru), la versión galesa, gestionada por la Welsh Authority, dirigida a la audiencia de Gales y a promocionar la cultura y la lengua de esa región.

El sistema de financiación de Channel 4 es un sistema mixto, en que se combinan, por un lado, un porcentaje de las aportaciones que reciben los poderes públicos por las licencia de las empresas privadas de televisión y, por otro, mediante publicidad ${ }^{136}$.

\section{RADIOTELEVISIÓN PRIVADA}

Por lo que a las radiotelevisiones privadas se refiere, como en la mayoría de los sistemas duales de radiotelevisión, se trata de empresas que han obtenido la correspondiente concesión o autorización. La radiotelevisión privada se rige por la Communications Act de 2003, que se encarga de establecer las condiciones para la obtención de licencias de radio y televisión así como de fijar una serie de medidas en relación a los contenidos ${ }^{137}$.

La primera televisión privada, la Independent Television (ITV), comenzó a emitir en 1956 y, aunque se financiaba con publicidad, como una empresa privada, se organizó de acuerdo a los principios de servicio público ${ }^{138}$. En ese primer momento, todas las televisiones, tanto públicas como privadas, tenían la obligación de prestar el servicio público de televisión, con independencia de sus fuentes de financiación ${ }^{139}$. Sin embargo, con la Broadcasting Act de 1991 se produjo una cierta liberalización de los servicios de televisión, con el fin de facilitar una mayor competencia. Así, el denominado tercer canal (Channel 3), la ITV (Independent Television) y el Channel 5, creado en 1995, tenían la obligación de prestar el servicio público de televisión, al ser televisiones que emitían por ondas terrestres, mientras que los canales que emitían por cable o por vía satélite, como BskyB, otro de los grandes grupos privados de comunicación del Reino Unido, no tenían esas obligaciones derivadas de la prestación del servicio público, aunque todas

135 Anteriormente estaba sometido a la Independent Televisión Commission, pero en la actualidad, estas tareas las desarrolla la OFCOM. Vid. Broadcasting Act de 1990, arts. 23 a 27. P. CAREy, Media Law, 2007, op. cit., op. cit., p. 254.

136 G. Pavani, «La Office of Communications (OfCOM) como autoridad independiente en el Reino Unido», op. cit., p. 204; T. GibBons «La televisión pública en el Reino Unido», op. cit., p. 53.

137 En relación con la adjudicación de licencias de televisión, vid. Communications Act 2003, secciones 211 a 244; en relación con la adjudicación de las licencias de radio, vid. Communications Act 2003, secciones 245 a 262 y en relación con los contenidos y las obligaciones de servicio público a las que están sometidos los medios privados de radio y televisión, vid. Communications Act 2003, capítulo IV.

138 A. Briggs, The history of Broadcasting in the UK, op. cit., p. 397 y ss.; T. GibBons, «La televisión pública en el Reino Unido», op. cit., p. 27; R. Craufurd SMITH, Broadcasting Law and Fundamental Rights, op. cit., p. 38.

139 G. Pavani, «La Office of Communications (OfCOM) como autoridad independiente en el Reino Unido», op. cit., p. 203. 
ellas, con independencia de la modalidad de difusión están obligadas a cumplir con ciertos estándares básicos en relación con los contenidos ${ }^{140}$.

En la actualidad, y debido a la evolución de las modalidades de transmisión de la señal producida en los últimos años, especialmente por la televisión digital terrestre, se han incrementado en el Reino Unido las plataformas de televisión de pago, además de multiplicarse la oferta de canales, entre los que destacan dos grandes empresas privadas de comunicación: ITV y BSkyB.

Como acabamos de indicar, las cadenas de radio y televisión privada, en el desarrollo de su actividad, quedan sujetas, en primer lugar, al cumplimiento de las normas sobre programación, en las que se fijan como principios el respeto al pluralismo, la objetividad, la veracidad y la igualdad de oportunidades, así como normas relativas a la protección de los menores de edad respecto de ciertos contenidos ${ }^{141}$. En segundo lugar, las radiotelevisiones privadas deben cumplir con las normas anticoncentración, para evitar que determinados grupos empresariales lleguen a ocupar una posición dominante en el mercado contraria al pluralismo. No obstante, estas normas anticoncentración no han evitado la creación de poderosos grupos de comunicación en el mercado audiovisual británico ${ }^{142}$. Además, se siguen manteniendo las obligaciones de servicio público, tanto para ITV (Channel 3) como para Channel $5^{143}$.

El control de las radios y televisiones privadas se encomienda a la OFCOM, autoridad independiente que desde 2002 desempeña las funciones que previamente realizaban la Independent Television Commission (ITC) en relación con las televisiones privadas ${ }^{144}$, y la Radio Autority en relación con las emisoras de radio privadas ${ }^{145}$.

\section{LAS «AUTORIDADES DE CONTROL»}

Hasta 2003 la organización y la regulación de la industria de la comunicación en el Reino Unido estaba muy fragmentada y existían autoridades de control específicas para cada uno de los sectores ${ }^{146}$. Así, la Independent Television Commis-

140 Así se establece en la Communications Act, Capítulo IV.

141 Communications Act, sección 319 y ss.

142 Communications Act, sección 348 y ss. En la doctrina, E. BAREndt, Broadcasting Law. A Comparative Study, 1993, pp. 28-29; J.D. Zelezny, Communications Law. Liberties, Restraints and the Modern Media, 2. ed., Wadsworth Publishing Company, 1997, p. 404 y ss. A. PÉrez GómEz, El control de las concentraciones de medios de comunicación. Derecho español y comparado, op. cit., p. 216 y ss.

143 Communications Act, sección 264.

144 La ITC ejercía competencias relacionadas con la adjudicación de licencias así como con la regulación del sector, en especial, respecto de la televisión comercial y los servicios por cable y por satélite. Además, tenía su propio código de conducta, el ITC Programme Code, al que se sometían todas las televisiones privadas, que fijaba los estándares en materia de contenidos, y cuyo incumplimiento podía dar lugar a sanciones económicas. S. RAGONE, «La autorregulación de los medios de comunicación en el Reino Unido», op. cit., p. 144.

$145 \mathrm{La}$ Radio Authority otorgaba las licencias a las radios comerciales nacionales y locales y regulaba los programas y la publicidad, además de tener potestad sancionadora.

146 G. PAVANi, «La Office of Communications (OFCOM) como autoridad independiente en el Reino Unido», en J. C. GAvara DE CARA (ed.), Las autoridades independientes de control de los medios de comunicación 
sion (ITC) y la Radio Authority tenían competencia en la concesión de licencias y en el control de la propiedad de las cadenas de radio y televisión privadas ${ }^{147}$. La Broadcasting Standards Commission (BSC) se encargaba de supervisar y resolver las quejas de los usuarios sobre contenidos inapropiados, tanto en los medios públicos como privados ${ }^{148}$. La Office of Telecommunications (OFTEL) era el órgano regulador de las telecomunicaciones y la Radiocommunications Agency era un organismo encargado de asignar las frecuencias en el espacio electromagnético.

Sin embargo, y con la entrada en vigor de la Communications Act de 2003 se creó una nueva autoridad audiovisual independiente, la Office of Communications (OFCOM), que ha asumido todas las funciones en materia audiovisual y de telecomunicaciones ${ }^{149}$. De este modo, la OFCOM se convierte en el «súper regulador», un organismo con competencias en materia de radio y televisión, tanto pública como privada, telecomunicaciones y otos servicios de comunicación (postal, inalámbrica, por ondas, etc. $)^{150}$.

Por otro lado, y en relación con la prensa, hasta 2014 la prensa escrita se sometía al control de la Press Complaints Commission (PCC), pero en esa fecha fue sustituida por la Independent Press Standard Organisation (IPSO).

\section{La Office of Communications (OFCOM)}

\section{A) Naturaleza}

La OFCOM es un organismo público con personalidad jurídica propia. Goza de autonomía financiera y presupuestaria y además, tiene autonomía administrativa interna y potestad reglamentaria.

Despliega sus competencias sobre todos los medios de comunicación audiovisuales del Reino Unido, tanto públicos como privados, independientemente de

audiovisual, Ed. Bosch, Barcelona, 2014, pp. 206 y 207.

147 Broadcasting Act de 1990, sección 2 (1) y 2 (2). La ITC tenía importantes competencias, ya que en el sector de la televisión privada se encargaba de la asignación de las licencias de televisión, controlaba los contenidos y la programación, así como el respeto a las normas anticoncentración, además de potestad sancionadora.

148 La BSC fue creada por la Broadcasting Act de 1996 y se constituyó como una autoridad de regulación y control en materia de programación y contenidos de las cadenas de televisión, públicas y privadas, además de resolver las reclamaciones presentadas por los particulares al respecto. Broadcasting Act de 1996, parte V, secciones 106 y ss. y Schedule 3, con disposiciones suplementarias sobre la BSC. En la doctrina, P. CArey, Media Law, op. cit., p. 214; S. RobIllard, Television in Europe: Regulatory Bodies, European Institute for the Media, London, 1995, p. 255; A. Rallo Lombarte, Pluralismo informativo y Constitución, Tirant lo Blanch, Valencia, 2000, p. 293.

149 Communications Act de 2003, Part 1, secciones 1 a 31. S. Buckley/K. Duer/T. Mendel/S. O Siochru, Broadcasting, Voice and Accountability. A Public Interest Approach to Policy, Law and Regulation, op. cit., p. 158; P. CAReY, Media Law, op. cit., p. 242.

150 G. Pavani, «La Office of Communications (OFCOM) como autoridad independiente en el Reino Unido», en J. C. Gavara de CARA (ed.), Las autoridades independientes de control de los medios de comunicación audiovisual, Ed. Bosch, Barcelona, 2014, p. 206. 
la plataforma de difusión utilizada, así como sobre los contenidos difundidos por esos medios ${ }^{151}$.

B) Funciones y competencias

a) Funciones

En el ejercicio de sus funciones, la OFCOM ha de proteger los intereses de los ciudadanos y de los consumidores y promover la libre competencia, y para ello, ha de cumplir con una serie de objetivos, como asegurar un uso adecuado y óptimo del espacio radioeléctrico que permita la disponibilidad de un amplio catálogo de servicios de comunicaciones electrónicas, incluidos los servicios de transferencia de datos de alta velocidad; garantizar la calidad de los servicios de radio y televisión, además de supervisar el cumplimiento de las obligaciones de servicio público; asegurar el pluralismo en la concesión de licencias de radio y televisión privadas; y ofrecer una adecuada protección a los ciudadanos en este sector, en especial, a los menores ${ }^{152}$. Además, la OFCOM ha de actuar conforme a los principios de transparencia, responsabilidad, orientados siempre a la consecución del «best practice».

Como hemos indicado anteriormente, la OFCOM se ha convertido en un organismo «súper regulador» que ha asumido funciones en distintos sectores del sector audiovisual y de las telecomunicaciones y otros servicios electrónicos, por lo que aquí vamos a hacer referencia únicamente a las funciones y competencias relativas a la radiotelevisión, pública y privada.

En este sentido, la OFCOM tiene encomendada la regulación y el control de las emisiones privadas de televisión que se difunden en el Reino Unido, así como de las emisiones de la BBC y de la Welsh Authority. Es la encargada de otorgar las licencias de televisión a las empresas privadas asegurando que los servicios de éstas se reciban en todo el territorio británico, y que se respetan las normas de competencia; de asegurar que se cumplen los estándares en la programación; de regular la publicidad; de garantizar los derechos de los ciudadanos en este sector; y de controlar el cumplimiento de las normas y, en su caso, de aplicar las sanciones que correspondan ${ }^{153}$.

Entre las funciones que lleva a cabo la OFCOM destacan la función reguladora, la potestad reglamentaria, la asignación de frecuencias y licencias, el control

151 A. EstradA, «La supervisión del pluralismo informativo en la radio y la televisión en Europa», en Cuadernos del CAC, n. ${ }^{\circ} 23$, p. 23 y ss; I. Katsirea, Public broadcasting and European Law. A comparative examination of public service obligations in six Member States, op. cit., p. 128 y ss.

152 Communications Act de 2003, sección 3 (2). G. Robertson/A. Nicol, Media Law, 5. a ed., Thomson, Sweet \& Maxwell, London, 2007, p. 884; G. PAVANI, «La Office of Communications (OFCOM) como autoridad independiente en el Reino Unido», op. cit., p. 213.

153 Communications Act de 2003, sección 198 y ss. y 211 y ss. 
sobre contenidos, la garantía de derechos fundamentales, en especial, los que tienen que ver con la esfera personal (honor, intimidad, secreto de las comunicaciones, protección de datos personales) y la potestad sancionadora ${ }^{154}$.

b) Competencias

a) Función reguladora - potestad reglamentaria: La OFCOM es la encargada de desarrollar lo establecido en la Communications Act, mediante la elaboración de normas de carácter reglamentario. En este sentido, destaca la labor de la OFCOM en la regulación de los planes de frecuencias y otros aspectos técnicos relacionados con los servicios de radiodifusión y la normativa en materia de programación, mediante la elaboración del denominado Broadcasting Code, en el que se fijan determinadas reglas en relación con los contenidos de los programas que han de cumplir todos los medios de radio y televisión, tanto públicos como privados ${ }^{155}$.

b) Concesión y renovación de licencias: La OFCOM gestiona y concede las licencias de uso del espectro radioeléctrico del Reino Unido, así como las licencias para todos los tipos de servicios de comunicación. El procedimiento y las condiciones para la concesión de estas licencias varían en función del tipo de servicio que se vaya a proporcionar ${ }^{156}$. En todo caso, las licencias se conceden a quienes reúnan las mejores condiciones, de acuerdo con la ley, y asegurando la libre competencia ${ }^{157}$.

En materia audiovisual, la OFCOM se encarga de la concesión de licencias a las cadenas de radio y televisión privada, además de la renovación de las mismas. En este sentido, todo lo relativo al procedimiento de concesión de licencias de radio y televisión, los requisitos que se establecen, las restricciones, etc, se regulan con detalle en la Communications Act y han sido desarrollados por la OFCOM ${ }^{158}$.

c) Control sobre los contenidos: La OFCOM tiene importantes competencias en relación con los contenidos de los programas, tanto de las televisiones públicas como de las privadas ${ }^{159}$. En cuanto al control del cumplimiento de las normas de programación, la OFCOM se encarga de elaborar un código de programación, denominado Broadcasting Code, en

154 En la actualidad, la OFCOM desarrolla más de 260 tareas en función del sector de actividad en el que esté actuando. Vid., al respecto, http://www.ofcom.org.uk/about/what-is-ofcom/

155 Communications Act de 2003, sección 69. S. Robillard, Television in Europe. Regulatory bodies, op. cit., p. 257; T. GibBons, Media Law in the United Kingdom, Wolters Kluwer, Leiden, 2012, p. 142 y ss.

156 G. PAVANI, «La Office of Communications (OFCOM) como autoridad independiente en el Reino Unido», op. cit., pp. 214 y 125.

157 Communications Act de 2003, sección 232 y ss.

158 Broadcasting Act de 1990, Parte 1. P. CArey, Media Law, op. cit., p. 210.

159 Communications Act de 2003, sección 263 y ss. 
el que se contienen los estándares sobre el contenido de los programas y que regula, entre otras, cuestiones como la obligación de respetar el buen gusto y la decencia, la imparcialidad y veracidad en la difusión de noticias, la protección de los menores, el tratamiento de la violencia, el respeto a la intimidad, las técnicas de recogida de información, la cobertura de la campaña electoral y el tratamiento de las noticias políticas, o las emisión de programas de contenido religioso ${ }^{160}$. Los principios contenidos en este código han de ser respetadas por todos los medios de comunicación audiovisual, tanto públicos como privados, aunque el incumplimiento de estas normas tiene sanciones de diferente intensidad dependiendo si el medio infractor es público o privado, ya que, en este último caso, puede llegar a suponer la no renovación de la licencia. Además, la OFCOM también se encarga de verificar el cumplimiento de determinados principios adicionales que la ley exige a determinados medios de comunicación, principalmente a los que desarrollan la función de servicio público ${ }^{161}$.

Por otro lado, la OFCOM tiene también competencias en relación con la publicidad y el patrocinio en las televisiones privadas. Por lo que a la publicidad se refiere, la OFCOM elabora un código (Code on the Scheduling of $T V$ advertising), en el que se regula todo lo relativo a los espacios publicitarios, como, por ejemplo, el tiempo máximo que se puede dedicar a publicidad, el intervalo de tiempo que tiene que transcurrir entre un bloque de espacios de publicidad y el siguiente, etc. ${ }^{162}$.

d) Facultades de control: La OFCOM es la encargada de controlar el cumplimiento por parte de las televisiones, tanto públicas como privadas de todas las normas que les son aplicables, en especial, las disposiciones de la Directiva Televisión sin Fronteras y el Convenio Europeo de Televisión Transfronteriza. Las competencias de control son especialmente importantes en relación con el cumplimiento de las normas de programación - tanto para las televisiones públicas como privadas - y de las normas publicidad y sobre concentración empresarial, en el caso de las televisiones privadas ${ }^{163}$.

e) Potestad sancionadora: La OFCOM tiene atribuida potestad sancionadora que ejerce de acuerdo con lo establecido en la ley ${ }^{164}$. En este sentido, la

160 Communications Act de 2003, sección 319. G. Robertson/A. Nicol, Media Law, op. cit., p. 887 y ss. http://stakeholders.ofcom.org.uk/broadcasting/broadcast-codes/

161 En este sentido, por ejemplo, a Channel 4 se le exige que financie o produzca programas educativos (Communications Act de 2003, secciones 295 a 297). También hay reglas propias para Channel 3 y Channel 5 (televisiones privadas que también tienen obligaciones de servicio público); Vid Communications Act de 2003, secciones 290 a 294 y 266 y 267 , respectivamente.

162 http://stakeholders.ofcom.org.uk/broadcasting/broadcast-codes/advert-code/

163 Communications Act de 2003, sección 263 y ss.

164 Communications Act de 2003, secciones 344 y ss. 
OFCOM desarrolla su potestad sancionadora especialmente en materia de contenidos. Así, puede sancionar a las televisiones si éstas incumplen con los valores del buen gusto, la decencia, la imparcialidad o la objetividad ya sea en los programas difundidos como en la publicidad (en el caso de las televisiones privadas).

La sanción puede consistir en: la exigencia de subsanación de un incumplimiento concreto (por ejemplo, la repetición de un programa, o la obligación de emitir rectificaciones o disculpas); la imposición de sanciones pecuniarias (en el caso de las televisiones privadas) ${ }^{165}$; la suspensión temporal de la licencia o incluso la revocación definitiva de la misma.

\section{D) Organización interna}

La estructura organizativa de la OFCOM es muy compleja debido a la función que desarrolla y a las muchas competencias que tiene atribuidas. Consta de dos órganos principales, la Junta Directiva o Board y el Comité Ejecutivo, además de numerosos consejos y comités, que desempeñan funciones principalmente consultivas y de asesoramiento a la Junta Directiva ${ }^{166}$.

\section{a) La Junta Directiva (Board)}

La Junta Directiva o Board está formada por un Presidente, un Vicepresidente y un máximo diez miembros nombrados por el Secretario de Estado de Cultura, Medios de Comunicación y Deporte. De entre los miembros que componen la Junta Directiva, uno de ellos ostenta la representación de Inglaterra, otro de Escocia, otro de Gales y otro de Irlanda del Norte ${ }^{167}$. En este sentido, debemos resaltar que un sector de la doctrina ha cuestionado el grado de independencia de la OFCOM, teniendo en cuenta que el nombramiento de los miembros corresponde al Gobierno ${ }^{168}$. Aun así, y con el fin de de asegurar que los nombramientos sean de acuerdo a circunstancias objetivas y por mérito y capacidad y no por cuestiones partidistas, para elegir a todos los miembros se deben respetar los principios establecidos por el Committee on Standars in Public Life, más conocidos como los «principios Nolan» ${ }^{169}$.

165 Estas sanciones recaen sobre los ingresos publicitarios, y oscilan entre un $3 \%$ del total de ingresos publicitarios, en caso del primer incumplimiento, y de un $5 \%$ en el caso de incumplimientos posteriores.

166 G. PaVAnI, «La Office of Communications (OFCOM) como autoridad independiente en el Reino Unido», op. cit., p. 208.

167 Communications Act de 2003, sección 12.

168 En este sentido, vid. E. BARENDT, Broadcasting Law, p. 83 y ss.

169 www.public-standards.gov.uk 
La Ley establece que no pueden ocupar un puesto como miembros de la OF$\mathrm{COM}$ quienes incurran en alguna de las siguientes causas de incompatibilidad: ser Trustee o empleado de la BBC; ser miembro de la Welsh Authority o ser miembro de la C4C. El mandato de los miembros de la Junta Directiva de la OFCOM tiene una duración máxima de cinco años y puede ser prorrogado. Lo relativo a la toma de posesión del cargo y su cese se determina en el nombramiento, con independencia de que cualquier miembro de la Junta Directiva de la Comisión pueda dimitir de su cargo, mediante escrito dirigido al Secretario de Estado ${ }^{170}$.

La Junta Directiva asume las más importantes funciones de dirección de la OFCOM y además puede crear comités o comisiones formadas por uno o varios de sus miembros para que, bajo su dirección, desempeñen algunas de sus funciones ${ }^{171} \mathrm{o}$ bien realicen tareas asesoras ${ }^{172}$.

b) El Comité Ejecutivo

El Comité Ejecutivo (Executive Commitee) se encarga de cuestiones ejecutivas y de management, además de cuestiones financieras y gestión administrativa de la entidad. Está subordinado a la Junta Directiva, de la que depende.

d) Los Consejos asesores

Para el desarrollo de las funciones que tiene atribuidas, la OFCOM cuenta con el apoyo de diferentes consejos asesores o consultivos.

Entre ellos, destaca el Comité Regional (Nations Committee), que tiene como función actuar de portavoz de los intereses de las sedes territoriales y que a su vez está formado por por los representantes de los cuatro consejos asesores regionales (Advisory Committees). Además, existen 10 Consejos Regionales de Telespectadores (Viewer Consultative Councils), el Consejo Asesor de Publicidad (Advertising Advisory Council) y el Comité Asesor Religioso (Central Religious Advisory Committee) $)^{173}$.

170 Communications Act de 2003, sección 12.9 y 12.12.

171 Entre los primeros, destacan el Operations Board, que a su vez se subdivide en diferentes áreas (área financiera, recursos humanos, organización y programación, etc.), el Content Board, que se encarga de cuestiones relacionadas con los contenidos audiovisuales; el Content Sanctions Committee, comité sancionador en materia de contenidos audiovisuales, el Comité de Licencias de Radio (Radio Licensing Comittee), el Fairness Committee, o el Election Committee, etc.

172 Por ejemplo, the Advisory Committee on Older and Disabled People, the Advisory Committee for the Nations, etc.

173 Communications Act de 2003, sección 20. S. Robillard, Television in Europe. Regulatory bodies, op. cit., p.260. 
e) Funcionamiento

En cuanto a su funcionamiento, la OFCOM tiene autonomía para establecer sus normas de funcionamiento interno.

Además, se organiza de forma descentralizada, a través de una sede central y las sedes territoriales (Advisory Committees) de las regiones integrantes del Reino Unido: Inglaterra, Gales, Escocia e Irlanda del Norte ${ }^{174}$. Los responsables de cada de una de estas sedes forman parte de la Junta directiva de la OFCOM. Además, se ha creado el Nations Committee, que constituye una comisión de la Junta Directiva y que tiene como función actuar de portavoz de los intereses de las sedes territoriales.

Por otro lado, OFCOM disfruta también de autonomía en la contratación y gestión del personal. Puede contratar a todos los empleados que considere necesarios y fija ella misma las condiciones laborales y salariales ${ }^{175}$.

\section{D) Financiación}

La OFCOM se financia principalmente a través de una tasa que deben abonar todos aquellos que han obtenido una licencia, ya sea de radio, televisión, servicios de telecomunicaciones o cualquier otro tipo de servicio de comunicación. La cuantía de la tasa varía dependiendo del tipo de licencia y se fija por propia Comisión, que puede, incluso, incrementar la cuantía de la misma para hacer frente a los costes de gestión de cada sector regulado. Además, también recibe financiación pública a través del HM Treasury, para el desarrollo de determinadas actividades, como, por ejemplo, cubrir los costes de la gestión del espacio electromagnético o para entablar contacto con otros organismos internacionales ${ }^{176}$.

Por otro lado, y teniendo en cuenta que los ingresos de la Comisión deben ser suficientes para que ésta pueda cumplir con sus obligaciones y desempeñar las tareas que le corresponden, la OFCOM puede recibir también, pagos anticipados (una especie de préstamo) abonados por el Parlamento. Estos pagos anticipados, que en todo caso tienen carácter excepcional, nunca podrán exceder de la cantidad de 5 millones de libras esterlinas y deberán ser reintegrados al Secretario de Estado junto con los intereses generados ${ }^{177}$.

174 G. Pavani, «La Office of Communications (OFCOM) como autoridad independiente en el Reino Unido», op. cit., p. 209.

175 Communications Act de 2003, sección 27

176 Communications Act de 2003, sección 399 y ss.; G. PAVAni, «La Office of Communications (OFCOM) como autoridad independiente en el Reino Unido», op. cit., p. 211.

177 Communications Act de 2003, sección 340. 
La Comisión debe presentar al final de cada año fiscal un presupuesto al Secretario de Estado, está obligada a llevar una contabilidad apropiada, y debe presentar las cuentas anuales a auditores independientes para su control ${ }^{178}$.

\section{E) Controles}

La OFCOM está obligada a presentar al Secretario de Estado un informe anual detallado en el que se recojan los datos de su actuación y en el que se incluya su presupuesto. Este informe es remitido posteriormente al Parlamento ${ }^{179}$.

Por otro lado, sus decisiones son recurribles ante los tribunales ${ }^{180}$.

\section{La Independent Press Standards Organisation (IPSO)}

En la actualidad, la prensa escrita en el Reino Unido se somete al control de la Independent Press Standards Organisation, que recientemente ha reemplazado a la Press Complaints Commission. La IPSO es un organismo de autorregulación independiente creado por la propia industria de la prensa en septiembre de 2014 y que supervisa la actuación de periódicos y revistas así como las versiones on-line de éstos. Este organismo está formado por la Junta Directiva, compuesta por doce miembros, incluido el Presidente, todos ellos profesionales del sector y por el Comité de Quejas (Complaints Committee), formado igualmente por doce miembros, en el que también participa el Presidente.

Respecto a su naturaleza jurídica, y al igual que ocurría con la PCC ${ }^{181}$, la IPSO no se considera un organismo regulador, ya que ninguna ley le ha habilitado para ello. Es un ente privado, que no ejerce potestades públicas, sino que únicamente se limita a actuar de mediador entre los medios y los ciudadanos y hacer cumplir los principios fijados en el Code of Practice elaborado por la propia industria de la prensa.

La IPSO desarrolla dos funciones principales. La primera, supervisar la práctica periodística, mediante el cumplimiento del Editor's Code of Practice. Este código se aplica al contenido de los periódicos y también a la actividad de los periodistas, que deben cumplir con los principios que se establecen en el mismo tanto

178 Communications Act de 2003, sección 399.

179 Communications Act de 2003, sección 390. G. PAVAnI, «La Office of Communications (OFCOM) como autoridad independiente en el Reino Unido», op. cit., p. 208.

180 G. Pavani, «La Office of Communications (OFCOM) como autoridad independiente en el Reino Unido», op. cit., p. 208.

181 G. Robertson/A. Nicol, Media Law, 4. ${ }^{a}$ Ed., Penguin Books, London, 2002, p. 684 (681-682); D. Bloy/S. Hadwin, Law and the media. For print, Broadcast and on line journalism, 2. ${ }^{a}$ ed., Sweet \& Maxwell, London, 2011, p. 369. 
en los medios de investigación utilizados como en la elaboración y contenido de las noticias que difunden. En este sentido, la IPSO tiene como objetivo lograr que se respeten unas normas éticas básicas que consigan equilibrar la protección de los derechos individuales y la libertad de prensa. El Code of Practice es revisado permanentemente para su actualización y a él se someten tanto los periodistas que trabajan para la prensa escrita (periódicos y revistas) como los de las versiones electrónicas $^{182}$.

La segunda de las funciones de la IPSO consiste en resolver o, al menos, adoptar las medidas pertinentes, en relación con las quejas o reclamaciones que cualquier ciudadano les haya remitido sobre una noticia que les afecte o para denunciar ciertas prácticas periodísticas. Para ello, a la IPSO se le ha dotado de mayores facultades, incluyéndose la potestad sancionadora así como la capacidad para determinar la naturaleza de la medida a adoptar, o la extensión y la ubicación de la rectificación de la noticia objeto del conflicto. Además, y como medida de transparencia, la IPSO publica en su página web todas las reclamaciones resueltas $^{183}$.

Las decisiones de la IPSO son recurribles ante los tribunales de justicia. No obstante, y con independencia de haberse dirigido a la IPSO o no, cualquier ciudadano puede dirigirse directamente a los tribunales si lo considera conveniente, esto es, no es preciso acudir previamente a este organismo.

$$
* * *
$$

TITLE: Status of the media in the United Kingdom

ABSTRACT: This essay analyzes the legal framework for the media in the UK - press, radio and television both public and private, as well as the supervisory authorities - which is based on the principles of independence, impartiality and self-regulation, and whose model of public broadcasting (BBC) is the most relevant reference in public service broadcasting.

RESUMEN: El trabajo que aquí presentamos tiene por objeto el análisis del régimen jurídico de los medios de comunicación en el Reino Unido — prensa, radio y televisión, pública y privada, así como las autoridades de supervisión y control-, que se caracteriza por ser un sistema basado en los principios de independencia, imparcialidad y autorregulación, y cuyo modelo de radiotelevisión pública (la BBC) constituye una referencia en la prestación del servicio público de radiodifusión.

KEY WORDS: media; radio and television, $B B C$, supervisory authorities.

Palabras Clave: Medios de comunicación, radiotelevisión, BBC, autoridades de control.

FECHA DE RECEPCIÓN: 03.06.2015

FECHA DE ACEPTACIÓN: 29.07.2015

182 La última versión del Editor's Code of Practice es de marzo de 2015, https://www.ipso.co.uk/assets/82/EDITORS_CODE_A4.pdf. Sobre los contenidos del Code of Practice, vid. G. SuTTER/D. GOLDBERG, "The Media and Media Law», en D. Goldberg/G. Sutter/I. Walden, Media Law and Practice, Oxford University Press, Oxford, 2009, p. 11. S. RAGONE, «La autorregulación de los medios de comunicación en el Reino Unido», op. cit., pp. 140 y 141.

183 https://www.ipso.co.uk/IPSO/rulings/resolution-statements/index.html 
